Article

\title{
New Dammarane-Type Triterpenoid Saponins from Panax notoginseng Leaves and Their Nitric Oxide Inhibitory Activities
}

\author{
Fan Sun ${ }^{1,+}{ }^{\text {, Jingya Ruan }}{ }^{1,+}{ }^{+}$, Wei Zhao ${ }^{1}$, Ying Zhang ${ }^{2}$, Guilin Xiang ${ }^{3}$, Jiejing Yan ${ }^{2}$, \\ Mimi Hao ${ }^{2}{ }^{\oplus}$, Lijie $\mathrm{Wu}^{1}$, Yi Zhang ${ }^{1,2, *}$ and Tao Wang ${ }^{1,2, *}$ \\ 1 Tianjin Key Laboratory of TCM Chemistry and Analysis, Tianjin University of Traditional Chinese Medicine, \\ 10 Poyanghu Road, West Area, Tuanbo New Town, Jinghai District, Tianjin 301617, China; \\ sf18435165322@163.com (F.S.); Ruanjy19930919@163.com (J.R.); zhaowei126123@126.com (W.Z.); \\ wulj0816@163.com (L.W.) \\ 2 Institute of TCM, Tianjin University of Traditional Chinese Medicine, 10 Poyanghu Road, West Area, Tuanbo \\ New Town, Jinghai District, Tianjin 301617, China; zyingtzy@163.com (Y.Z.); 17320072093@163.com (J.Y.); \\ haomimi126@126.com (M.H.) \\ 3 WenshanMiaoxiangSanqi Limited Company, South KaihuaRoad, Wenshan 663000, China; \\ xiangguilin@126.com \\ * Correspondence: zhwwxzh@tjutcm.edu.cn (Y.Z.); wangtao@tjutcm.edu.cn (T.W.); \\ Tel./Fax: +86-22-5959-6168 (T.W.) \\ + These authors contributed equally to this work.
}

Academic Editor: Deok-Chun Yang

Received: 4 December 2019; Accepted: 26 December 2019; Published: 29 December 2019

\begin{abstract}
Inflammation is a very common and important pathological process that can cause many diseases. The discovery of anti-inflammatory drugs and the treatment of inflammation are particularly essential. Dammarane-type triterpenoid saponins (PNS) were demonstrated to show anti-inflammatory effects in the leaves of Panax notoginseng. Chromatographies and spectral analysis methods were combined to isolate and identify PNS. Moreover, the nitric oxide (NO) inhibitory activities of all compounds were examined in lipopolysaccharide (LPS)-stimulated RAW264.7 cells. As a result, eleven new dammarane-type triterpenoid saponins, notoginsenosides $\mathrm{NL}-\mathrm{A}_{1}-\mathrm{NL}-\mathrm{A}_{4}$ (1-4), NL-B $\mathrm{B}_{1}-\mathrm{NL}-\mathrm{B}_{3}(5-7), \mathrm{NL}-\mathrm{C}_{1}-\mathrm{NL}-\mathrm{C}_{3}(8-10)$, and NL-D (11) were isolated, and their structures were identified by using various spectrometric techniques and chemical reactions. Among them, compounds $\mathbf{4}$ and $\mathbf{1 1}$ were characterized by the malonyl substitution at 3-position. The 3-malonyl substituted dammarane-type terpennoids were first obtained from natural products. In addition, compounds 1, 2, 5, 6, and 8-10 were found to play an important role in suppressing NO levels at $50 \mu \mathrm{M}$, without cytotoxicity. All inhibitory activities were found to be dose-dependent.
\end{abstract}

Keywords: Panax notoginseng leaves; dammarane-type triterpenoid saponins; notoginsenosides NL; RAW 246.7 cell; anti-inflammatory activity

\section{Introduction}

Panax notoginseng (Burk.) F. H. Chen is generally known as San qi in Chinese. As recorded in Chinese medical book "Compendium of Material Medica", its root was traditionally used in Asia for the treatment of trauma, body pain, inflammation, and cardiovascular diseases since ancient times [1]. Due to the excellent medical property of $P$. notoginseng, medicines that contain this leaf are commercially available and are widely applied in clinics in China. For example, $P$. notoginseng was found to be the main ingredient in Xuesaitong injections and Xuesaitong capsules [2], Yun-Nan-Bai-Yao [3], as well as 
Xueshuantong injections and Xueshuantong capsules. As is known, the triterpenoid saponins in it largely contributes to the above-mentioned biological activities [2,3].

Since the ability of $P$. notoginseng to adapt to the environment declined gradually through hundreds of years of cultivation, the problem of continuous cropping became more and more prominent, which resulted in the decrease of P. notoginseng root yields [4]. On the other hand, the harvest of $P$. notoginseng root required long growth periods. Research has showed that $P$. notoginseng leaves are rich in dammarane-type triterpenoid saponins (PNS) [5-10], suggesting that the leaves could be a possible replacement of the roots. In order to expand the utilization of $P$. notoginseng resources, the development and application of its leaves have gradually attracted the attention and interest of scholars.

Inflammation is a very common and important pathological process that can cause many diseases [11]. The discovery of anti-inflammatory drugs and the treatment of inflammation are particularly essential. PNS were demonstrated to show anti-inflammatory effects in P. notoginseng [3].

Herein, chromatographies and spectral analysis methods were combined to isolate and identify PNS from P. notoginseng leaves. Moreover, the inhibitory activities of obtained PNS against nitric oxide (NO) production in RAW 264.7 cells induced by lipopolysaccharide (LPS) were measured.

\section{Results and Discussion}

The $50 \% \mathrm{EtOH}$ extract of $P$. notoginseng leaves was isolated by D101 macroporous resin column chromatography (CC), and was eluted with $\mathrm{H}_{2} \mathrm{O}$ and $95 \% \mathrm{EtOH}$, successively. The obtained 95\% EtOH eluate was separated by CCs such as silica gel, Sephadex LH-20, and preparative high-performance liquid chromatography (pHPLC), and eleven new dammarane-type triterpenoid saponins, notoginsenosides NL- $\mathrm{A}_{1}-\mathrm{NL}-\mathrm{A}_{4}$ (1-4), NL- $\mathrm{B}_{1}-\mathrm{NL}-\mathrm{B}_{3}$ (5-7), NL-C $\mathrm{C}_{1}-\mathrm{NL}-\mathrm{C}_{3}$ (8-10), and NL-D(11) (Figure 1) were yielded.

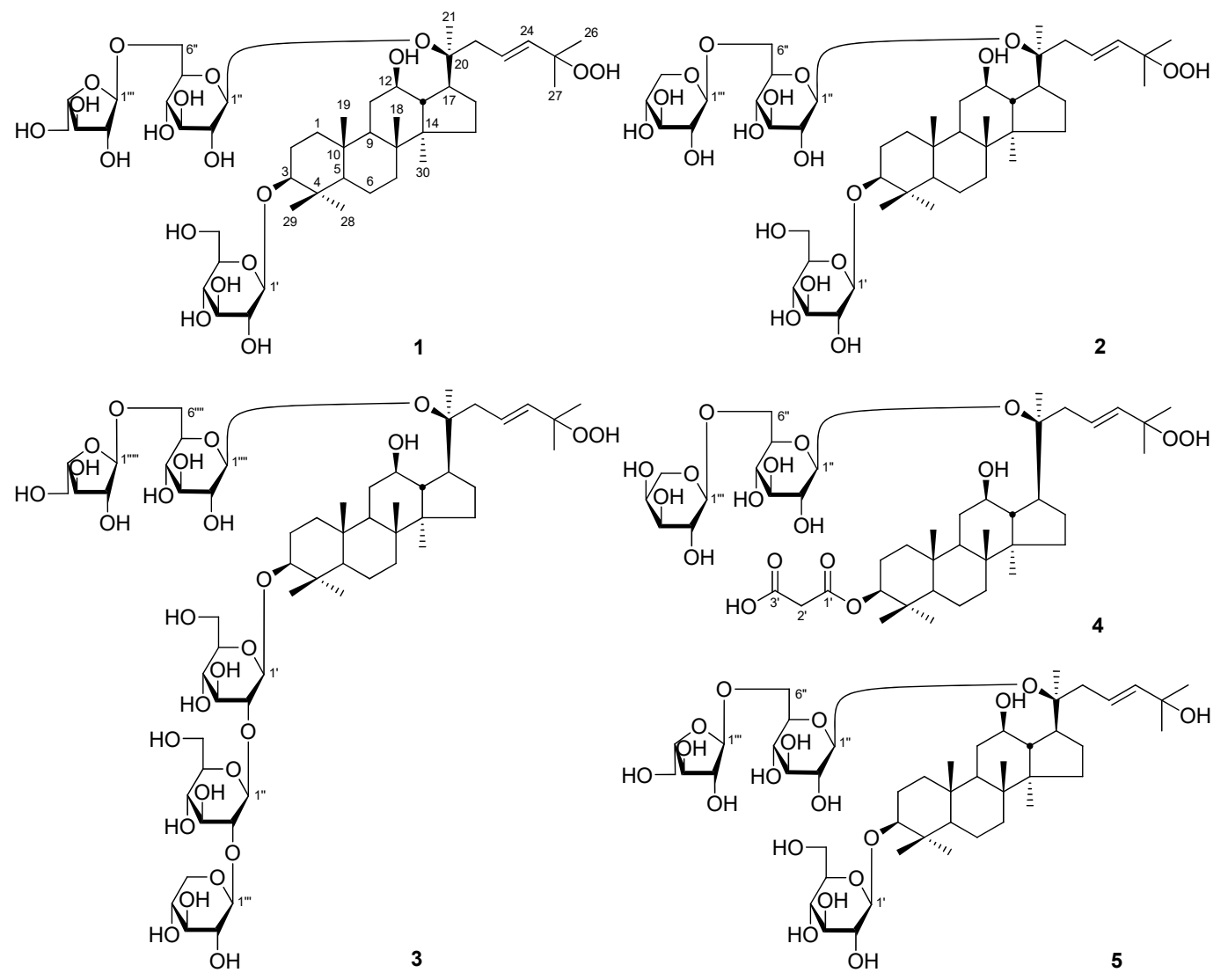

Figure 1. Cont. 

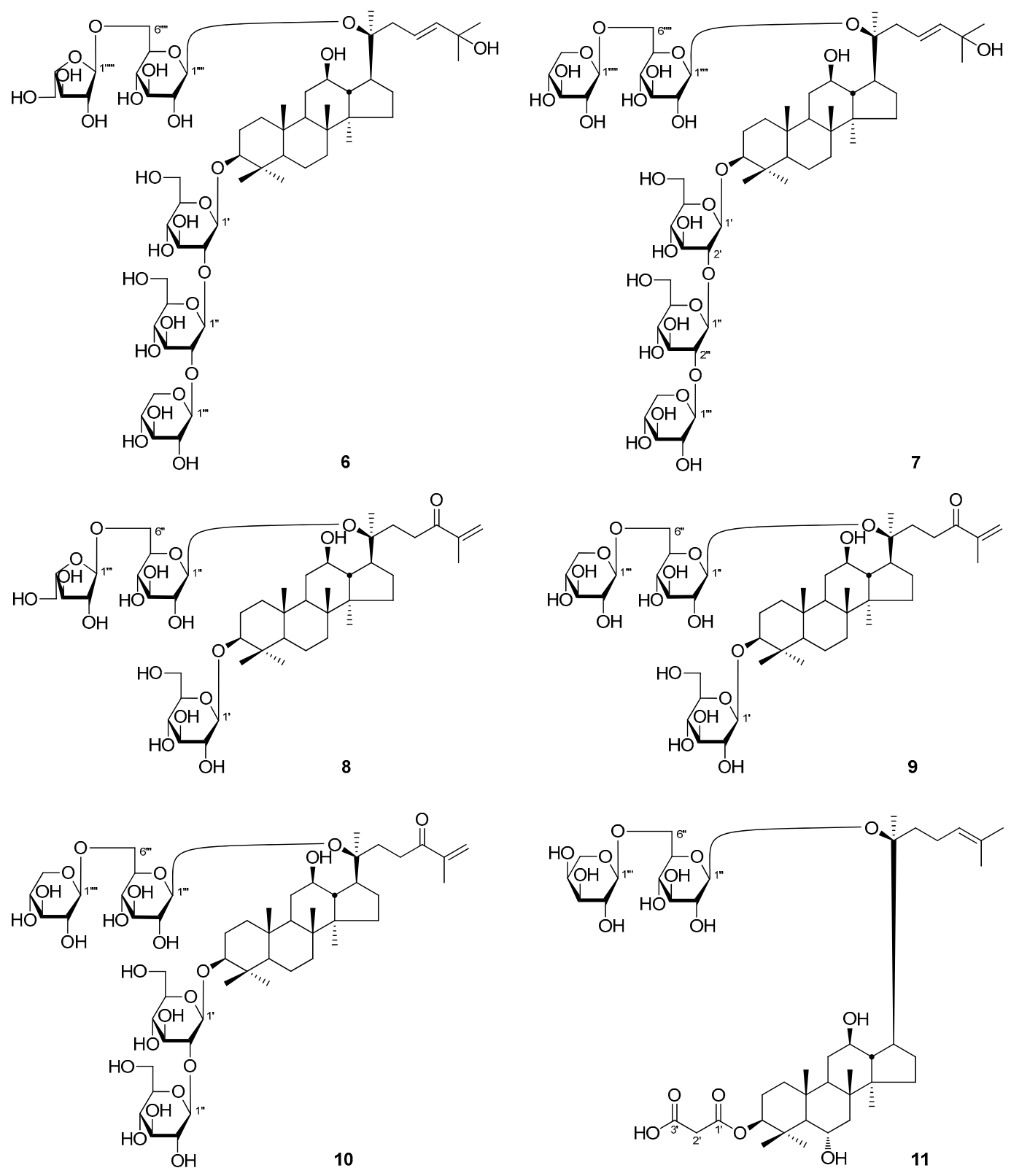

Figure 1. The new compounds 1-11 obtained from Panax notoginseng leaves.

Notoginsenoside NL-A 1 (1) was isolated as a white powder with a negative optical rotation $\left([\alpha]_{\mathrm{D}}^{25}-1.8, \mathrm{MeOH}\right)$. Its molecular formula, $\mathrm{C}_{47} \mathrm{H}_{80} \mathrm{O}_{19}\left(\mathrm{~m} / z \mathrm{z} 97.52405[\mathrm{M}-\mathrm{H}]^{-}\right.$; calcd. for $\mathrm{C}_{47} \mathrm{H}_{79} \mathrm{O}_{19}$, 947.52101) was measured on negative-ion ESI-Q-Orbitrap MS. The IR spectrum showed the absorption bands assignable to hydroxyl $\left(3395 \mathrm{~cm}^{-1}\right)$, olefin $\left(1645 \mathrm{~cm}^{-1}\right)$, and ether $\left(1078 \mathrm{~cm}^{-1}\right)$ functions, respectively. Acid hydrolysis of $\mathbf{1}$ followed by HPLC analysis confirmed the presence of D-glucose and L-arabinose [12]. The ${ }^{1} \mathrm{H}$ and ${ }^{13} \mathrm{C}-\mathrm{NMR}$ (Table 1) spectra of $\mathbf{1}$ displayed the signals of two $\beta$-D-glucopyranosyls [ $\left.\delta 4.95\left(1 \mathrm{H}, \mathrm{d}, J=8.0 \mathrm{~Hz}, \mathrm{H}-1^{\prime}\right), 5.18\left(1 \mathrm{H}, \mathrm{d}, J=8.0 \mathrm{~Hz}, \mathrm{H}-1^{\prime \prime}\right)\right]$, and one $\alpha$-L-arabinofuranosyl [ $\left.\delta 5.66\left(1 \mathrm{H}, \mathrm{d}, J=1.5 \mathrm{~Hz}, \mathrm{H}-\mathrm{C}^{\prime \prime \prime}\right)\right]$. Its ${ }^{13} \mathrm{C}-\mathrm{NMR}$ spectrum showed forty-seven signals. After subtracting the seventeen carbon resonances that belonged to the sugar units, the remaining thirty resonances were attributable to a triterpene skeleton. In the ${ }^{1} \mathrm{H}-\mathrm{NMR}$ spectrum, eight signals could be assigned to methyls [ $\delta 0.81,0.90,1.00,1.02,1.32$ (3H each, all s, $\mathrm{H}_{3}-19,30,29,18$, and 28), and $1.61\left(9 \mathrm{H}, \mathrm{s}, \mathrm{H}_{3}-21,26\right.$, and 27)], two signals belonged to oxygenated methylene [ $\delta 3.36$ $(1 \mathrm{H}, \mathrm{dd}, J=4.0,11.5 \mathrm{~Hz}, \mathrm{H}-3), 4.02(1 \mathrm{H}, \mathrm{m}, \mathrm{H}-12)]$, and the signals for one trans-olefin $[\delta 6.11(1 \mathrm{H}, \mathrm{d}$, $J=16.0 \mathrm{~Hz}, \mathrm{H}-24), 6.16(1 \mathrm{H}, \mathrm{ddd}, J=5.5,8.0,16.0 \mathrm{~Hz}, \mathrm{H}-23)]$ indicated that 1 was a dammarane-type 
triterpene saponin derivative. In order to solve the problem of overlapping for the three glycosyl groups, HSQC-TOCSY experiment was performed. In the HSQC-TOCSY spectrum, correlations were found between the following proton and carbon pairs: $\delta_{\mathrm{H}} 4.95\left(\mathrm{H}-1^{\prime}\right)$ and $\delta_{\mathrm{C}} 71.8\left(\mathrm{C}-4^{\prime}\right), 75.7$ $\left(\mathrm{C}-2^{\prime}\right), 78.7\left(\mathrm{C}-3^{\prime}\right), 107.1\left(\mathrm{C}-1^{\prime}\right) ; \delta_{\mathrm{H}} 4.42,4.62\left(\mathrm{H}_{2}-6^{\prime}\right)$ and $\delta_{\mathrm{C}} 63.0\left(\mathrm{C}-6^{\prime}\right), 71.8\left(\mathrm{C}-4^{\prime}\right), 78.4\left(\mathrm{C}-5^{\prime}\right) ; \delta_{\mathrm{H}}$ $5.18\left(\mathrm{H}-1^{\prime \prime}\right)$ and $\delta_{\mathrm{C}} 71.9\left(\mathrm{C}-4^{\prime \prime}\right), 75.1\left(\mathrm{C}-2^{\prime \prime}\right), 78.8\left(\mathrm{C}-3^{\prime \prime}\right), 98.2\left(\mathrm{C}-1^{\prime \prime}\right) ; \delta_{\mathrm{H}} 4.13,4.66\left(\mathrm{H}_{2}-6^{\prime \prime}\right)$ and $\delta_{\mathrm{C}} 68.3$ $\left(\mathrm{C}-6^{\prime \prime}\right), 71.9\left(\mathrm{C}-4^{\prime}\right), 76.4\left(\mathrm{C}-5^{\prime \prime}\right) ; \delta_{\mathrm{H}} 5.66\left(\mathrm{H}-1^{\prime \prime \prime}\right)$ and $\delta_{\mathrm{C}} 83.3\left(\mathrm{C}-2^{\prime \prime \prime}\right), 110.0\left(\mathrm{C}-1^{\prime \prime \prime}\right) ; \delta_{\mathrm{H}} 4.87\left(\mathrm{H}-2^{\prime \prime \prime}\right)$ and $\delta_{\mathrm{C}} 78.9\left(\mathrm{C}-3^{\prime \prime \prime}\right), 83.3\left(\mathrm{C}-2^{\prime \prime \prime}\right), 85.9\left(\mathrm{C}-4^{\prime \prime \prime}\right), 110.0\left(\mathrm{C}-1^{\prime \prime \prime}\right) ; \delta_{\mathrm{H}} 4.21,4.31\left(\mathrm{H}_{2}-6^{\prime \prime \prime}\right)$ and $\delta_{\mathrm{C}} 62.7\left(\mathrm{C}-5^{\prime \prime \prime}\right)$, $78.9\left(\mathrm{C}-3^{\prime \prime \prime}\right), 83.3\left(\mathrm{C}-2^{\prime \prime \prime}\right), 85.9\left(\mathrm{C}-4^{\prime \prime \prime}\right)$. In conjunction with the HSQC spectrum, the spectroscopic data of the above-mentioned three glycosyls were assigned. According to the proton and proton correlations observed in its ${ }^{1} \mathrm{H}-{ }^{1} \mathrm{H}$ COSY spectrum (Figure 2), seven moieties written in bold lines were denoted. Moreover, its planar structure of was clarified by the correlations from $\mathrm{H}_{3}-18$ to C-7-9, C-14; $\mathrm{H}_{3}-19$ to C-1, C-5, C-9, C-10; $\mathrm{H}_{3}-21$ to C-17, C-20, C-22; $\mathrm{H}_{3}-26$ and C-24, C-25, C-27; $\mathrm{H}_{3}-27$ to $\mathrm{C}-24-26 ; \mathrm{H}_{3}-28$ to $\mathrm{C}-3-5, \mathrm{C}-29 ; \mathrm{H}_{3}-29$ to $\mathrm{C}-3-5, \mathrm{C}-28 ; \mathrm{H}_{3}-30$ to $\mathrm{C}-8, \mathrm{C}-13-15 ; \delta_{\mathrm{H}} 4.95\left(\mathrm{H}-1^{\prime}\right)$ to $\delta_{\mathrm{C}} 88.8(\mathrm{C}-3) ; \delta_{\mathrm{H}} 5.18\left(\mathrm{H}-1^{\prime \prime}\right)$ to $\delta_{\mathrm{C}} 83.2(\mathrm{C}-20) ; \delta_{\mathrm{H}} 5.66\left(\mathrm{H}-1^{\prime \prime \prime}\right)$ to $\delta_{\mathrm{C}} 68.3\left(\mathrm{C}-6^{\prime \prime}\right)$ displayed in its HMBC spectrum. The resonance of $C-25$ were shifted downfield by about $11 \mathrm{ppm}$, as compared with notoginsenoside Fh5 with $3 \beta, 12 \beta, 20(S), 25$-tetrahydroxydammar-23-ene as aglycone [6], indicating that C- 25 of compound 1 was substituted by the hydroperoxyl group. Moreover, since the chemical shifts of notoginsenoside NL-A $A_{1}(\mathbf{1})$ aglycone were identical to those of the known compound, $3 \beta, 12 \beta, 20 S$-trihydroxy-25-hydroperoxydammar-23-ene-3-O-[ $\beta$-D-glucopyranosyl $(1 \rightarrow 2)-\beta$-D-glucopyranosyl] -20-O-[ $\beta$-D-xylopyranosyl $(1 \rightarrow 6)]-\beta$-D-glucopyranoside [13], its aglycone was determined to be $3 \beta, 12 \beta, 20 S$-trihydroxy-25-hydroperoxydammar-23-ene.

Table 1. The ${ }^{1} \mathrm{H}$ and ${ }^{13} \mathrm{C}-\mathrm{NMR}$ data for $\mathbf{1}$ in $\mathrm{C}_{5} \mathrm{D}_{5} \mathrm{~N}$.

\begin{tabular}{|c|c|c|c|c|c|}
\hline No. & $\delta_{C}$ & $\delta_{\mathrm{H}}(J$ in $\mathrm{Hz})$ & No. & $\delta_{C}$ & $\delta_{\mathrm{H}}(J$ in $\mathrm{Hz})$ \\
\hline 1 & 39.1 & $0.78,1.56$ (both m) & 25 & 81.3 & - \\
\hline 2 & 26.7 & $\begin{array}{c}1.81(\mathrm{~m} \text {, overlapped }) \\
2.21(\mathrm{~m})\end{array}$ & 26 & 25.4 & $1.61(\mathrm{~s})$ \\
\hline 3 & 88.8 & $3.36(\mathrm{dd}, 4.0,11.5)$ & 27 & 25.1 & $1.61(\mathrm{~s})$ \\
\hline 4 & 39.7 & - & 28 & 28.1 & $1.32(\mathrm{~s})$ \\
\hline 5 & 56.4 & 0.73 (br. d, ca. 12) & 29 & 16.8 & $1.00(\mathrm{~s})$ \\
\hline 6 & 18.4 & $\begin{array}{c}1.39(\mathrm{~m} \text {, overlapped }) \\
1.51(\mathrm{~m})\end{array}$ & 30 & 17.2 & $0.90(\mathrm{~s})$ \\
\hline 7 & 35.0 & $1.22,1.49$ (both m) & $1^{\prime}$ & 107.1 & $4.95(\mathrm{~d}, 8.0)$ \\
\hline 8 & 40.0 & - & $2^{\prime}$ & 75.7 & $4.06(\mathrm{~m}$, overlapped $)$ \\
\hline 9 & 50.1 & 1.39 (m, overlapped) & $3^{\prime}$ & 78.7 & $4.27(\mathrm{dd}, 8.0,9.0)$ \\
\hline 10 & 36.9 & - & $4^{\prime}$ & 71.8 & $4.22(\mathrm{~m})$ \\
\hline 11 & 30.7 & $\begin{array}{c}1.56(\mathrm{~m}) \\
2.01(\mathrm{~m}, \text { overlapped })\end{array}$ & $5^{\prime}$ & 78.4 & $4.03(\mathrm{~m}$, overlapped $)$ \\
\hline 12 & 70.6 & $4.02(\mathrm{~m})$ & $6^{\prime}$ & 63.0 & $\begin{array}{l}4.42(\mathrm{dd}, 5.5,11.5) \\
4.62(\mathrm{dd}, 2.0,11.5)\end{array}$ \\
\hline 13 & 49.4 & 2.01 (m, overlapped) & $1^{\prime \prime}$ & 98.2 & $5.18(\mathrm{~d}, 8.0)$ \\
\hline 14 & 51.5 & - & $2^{\prime \prime}$ & 75.1 & $3.97(\mathrm{dd}, 7.5,8.0)$ \\
\hline 15 & 30.5 & $\begin{array}{c}0.98(\mathrm{~m}) \\
1.61(\mathrm{~m}, \text { overlapped })\end{array}$ & $3^{\prime \prime}$ & 78.8 & $4.20(\mathrm{~m}$, overlapped $)$ \\
\hline 16 & 26.3 & $1.47,1.79$ (both m) & $4^{\prime \prime}$ & 71.9 & $4.03(\mathrm{~m}$, overlapped $)$ \\
\hline 17 & 52.0 & 2.44 (q like, ca. 11) & $5^{\prime \prime}$ & 76.4 & $4.09\left(\mathrm{~m}, \mathrm{H}-5^{\prime \prime}\right)$ \\
\hline 18 & 16.0 & $1.02(\mathrm{~s})$ & $6^{\prime \prime}$ & 68.3 & $\begin{array}{l}4.13(\mathrm{dd}, 5.0,11.0) \\
4.66 \text { (br. d, ca. 11) }\end{array}$ \\
\hline 19 & 16.3 & $0.81(\mathrm{~s})$ & $1^{\prime \prime \prime}$ & 110.0 & $5.66(\mathrm{~d}, 1.5)$ \\
\hline 20 & 83.2 & - & $2^{\prime \prime \prime}$ & 83.3 & 4.87 (br. s), \\
\hline 21 & 23.3 & $1.61(\mathrm{~s})$ & $3^{\prime \prime \prime}$ & 78.9 & $4.78(\mathrm{~m})$ \\
\hline 22 & 39.8 & $\begin{array}{l}2.81(\mathrm{dd}, 8.0,14.0) \\
3.08(\mathrm{dd}, 5.5,14.0)\end{array}$ & $4^{\prime \prime \prime}$ & 85.9 & $4.78(\mathrm{~m})$ \\
\hline 23 & 126.7 & $6.16(\mathrm{ddd}, 5.5,8.0,16.0)$ & $5^{\prime \prime \prime}$ & 62.7 & $\begin{array}{c}4.21 \text { (m, overlapped) } \\
4.31 \text { (br. d, ca. 11) }\end{array}$ \\
\hline 24 & 138.0 & $6.11(\mathrm{~d}, 16.0)$ & & & \\
\hline
\end{tabular}



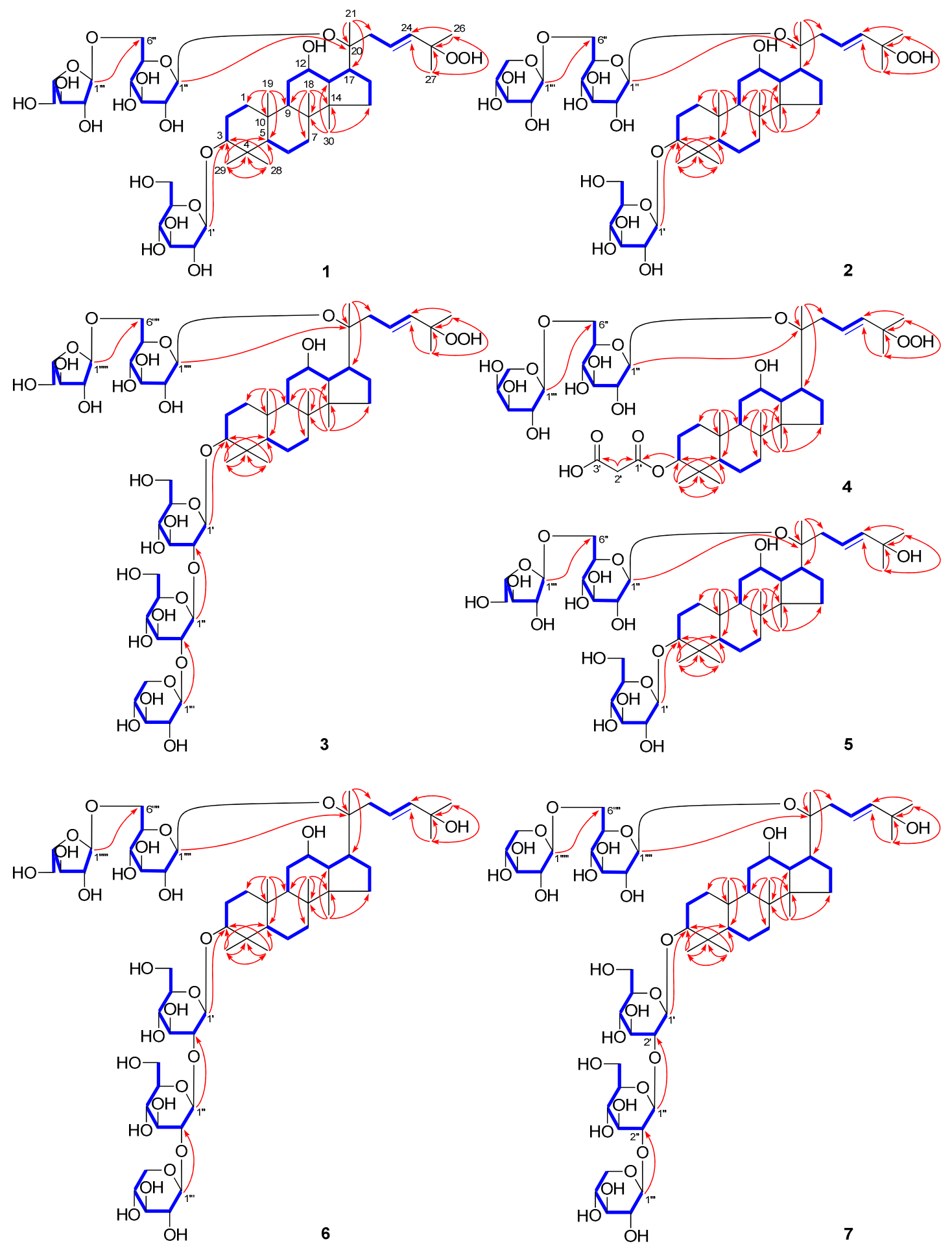

Figure 2. Cont. 

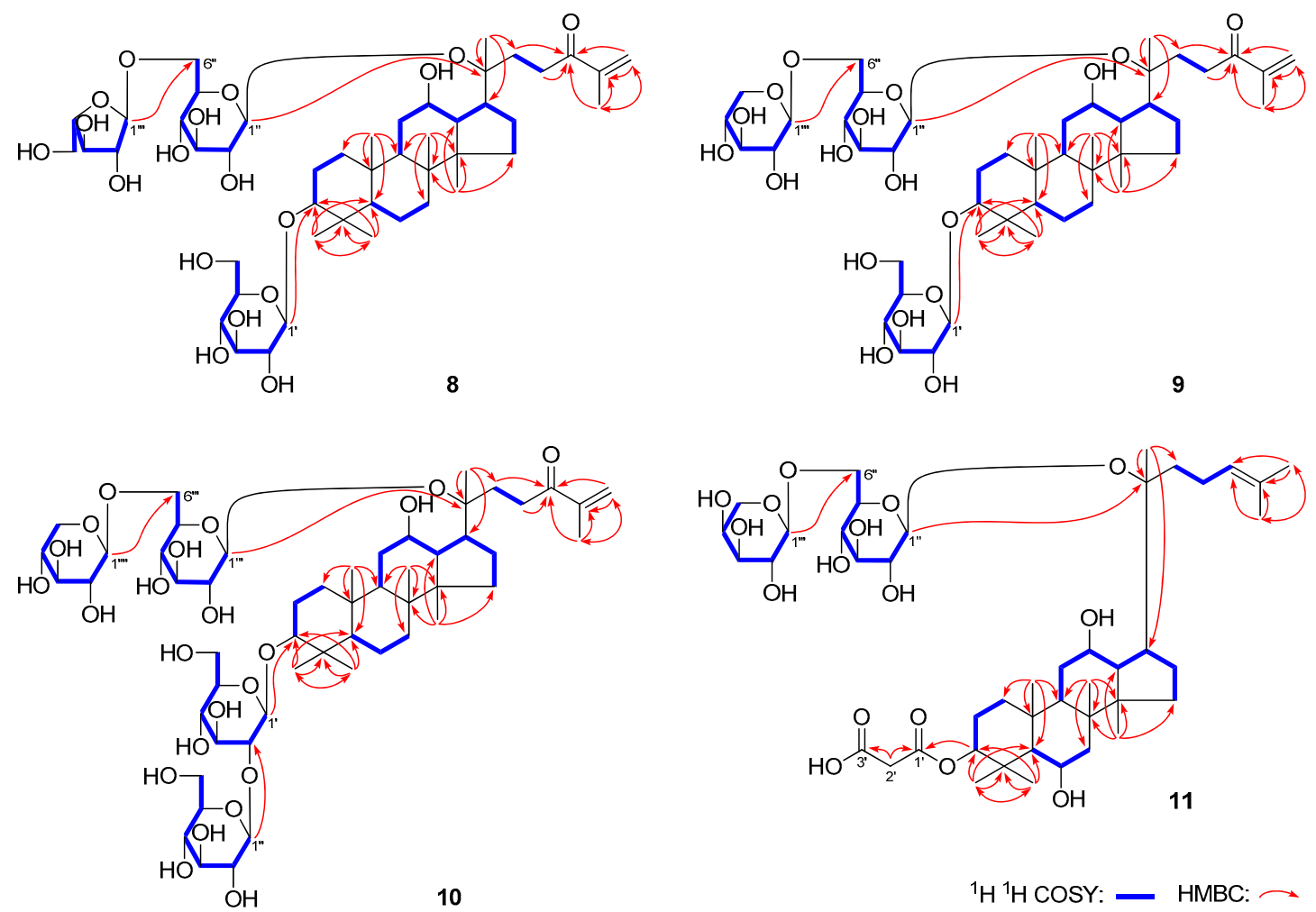

Figure 2. Key ${ }^{1} \mathrm{H}-{ }^{1} \mathrm{H}$ COSY and HMBC correlations of 1-11.

Both notoginsenosides $\mathrm{NL}-\mathrm{A}_{2}$ (2) and $\mathrm{NL}^{-\mathrm{A}_{3}}$ (3) were obtained as a white powder. The ${ }^{1} \mathrm{H}$ and ${ }^{13} \mathrm{C}-\mathrm{NMR}$ (Tables 2 and 3 ) and $2 \mathrm{D}-\mathrm{NMR}$ spectra, including ${ }^{1} \mathrm{H}-{ }^{1} \mathrm{H}$ COSY, HSQC, HSQC-TOCSY, and HMBC suggested 2 and 3 had the same aglycone, $3 \beta, 12 \beta, 20 S$-trihydroxy-25-hydroperoxydammar-23-ene as that of $\mathbf{1}$. Meanwhile, compound 2 possessed the same molecular formula, $\mathrm{C}_{47} \mathrm{H}_{80} \mathrm{O}_{19}\left(\mathrm{~m} / z 947.51996[\mathrm{M}-\mathrm{H}]^{-}\right.$; calcd. for $\mathrm{C}_{47} \mathrm{H}_{79} \mathrm{O}_{19}$, 947.52101) as 1. The main difference between $\mathbf{2}$ and $\mathbf{1}$ was that the $\alpha$-L-arabinofuranosyl group in $\mathbf{1}$ was replaced for $\beta$-D-xylopyranosyl group $\left[\delta 5.00\left(1 \mathrm{H}, \mathrm{d}, J=7.5 \mathrm{~Hz}, \mathrm{H}-1^{\prime \prime \prime}\right)\right]$ in 2 . As compared to compound 1, one more $\beta$-D-glucopyranosyl [ $\left.\delta 5.50\left(1 \mathrm{H}, \mathrm{d}, J=7.5 \mathrm{~Hz}, \mathrm{H}-1^{\prime \prime}\right)\right]$ and one more $\beta$-D-xylopyranosyl [ 5.39 $\left.\left(1 \mathrm{H}, \mathrm{d}, J=7.0 \mathrm{~Hz}, \mathrm{H}-1^{\prime \prime \prime}\right)\right]$ presented in 3, which was clarified by its ESI-Q-Orbitrap MS determination $(\mathrm{m} / z \text { 1241.61584 [M - H] }]^{-}$; calcd. for $\left.\mathrm{C}_{58} \mathrm{H}_{97} \mathrm{O}_{28}, 1241.61609\right)$. Meanwhile, as compared to $1, \delta_{\mathrm{C}} \mathbf{1}^{\prime}$ and $\delta_{C}-2^{\prime}$ of 3 were shifted upfield and downfield by $\left[\delta_{C} 75.7\left(C-2^{\prime}\right), 107.1\left(C-1^{\prime}\right)\right.$ for $1 ; \delta_{C} 82.9\left(C-2^{\prime}\right), 104.8$ $\left(\mathrm{C}-1^{\prime}\right)$ for 3], respectively, which suggested $\mathrm{C}-2^{\prime}$ in 3 might be substituted by the glycosyl group. The cross peaks from $\delta_{\mathrm{H}} 4.98\left(\mathrm{H}-1^{\prime}\right)$ to $\delta_{\mathrm{C}} 88.8(\mathrm{C}-3) ; \delta_{\mathrm{H}} 5.20\left(\mathrm{H}-1^{\prime \prime}\right)$ to $\delta_{\mathrm{C}} 83.2(\mathrm{C}-20) ; \delta_{\mathrm{H}} 5.00\left(\mathrm{H}-1^{\prime \prime \prime}\right)$ to $\delta_{\mathrm{C}}$ $69.9\left(\mathrm{C}-6^{\prime \prime}\right)$ were observed in 2 ; while the long range correlations from $\delta_{\mathrm{H}} 4.92\left(\mathrm{H}-1^{\prime}\right)$ to $\delta_{\mathrm{C}} 89.0(\mathrm{C}-3)$; $\delta_{\mathrm{H}} 5.50\left(\mathrm{H}-1^{\prime \prime}\right)$ to $\delta_{\mathrm{C}} 82.9\left(\mathrm{C}-2^{\prime}\right) ; \delta_{\mathrm{H}} 5.39\left(\mathrm{H}-1^{\prime \prime \prime}\right)$ to $\delta_{\mathrm{C}} 84.6\left(\mathrm{C}-2^{\prime \prime}\right) ; \delta_{\mathrm{H}} 5.17\left(\mathrm{H}-1^{\prime \prime \prime \prime}\right)$ to $\delta_{\mathrm{C}} 83.2(\mathrm{C}-20)$; $\delta_{\mathrm{H}} 5.64\left(\mathrm{H}-1^{\prime \prime \prime \prime \prime \prime}\right)$ to $\delta_{\mathrm{C}} 68.4\left(\mathrm{C}-6^{\prime \prime \prime \prime}\right)$ were found (Figure 2) in 3 . The structures of 2 and 3 were thus elucidated. Using methods similar as $\mathbf{1}$, the NMR data of each sugar moieties in $\mathbf{2}$ and $\mathbf{3}$ were assigned in detail. 
Table 2. The ${ }^{1} \mathrm{H}$ and ${ }^{13} \mathrm{C}-\mathrm{NMR}$ data for 2 in $\mathrm{C}_{5} \mathrm{D}_{5} \mathrm{~N}$.

\begin{tabular}{|c|c|c|c|c|c|}
\hline No. & $\delta_{C}$ & $\delta_{H}(J$ in $\mathrm{Hz})$ & No. & $\delta_{C}$ & $\delta_{H}(J$ in $\mathrm{Hz})$ \\
\hline 1 & 39.1 & $\begin{array}{c}0.78(\mathrm{~m}) \\
1.57(\mathrm{~m}, \text { overlapped })\end{array}$ & 25 & 81.4 & - \\
\hline 2 & 26.8 & $1.84,2.23$ (both m) & 26 & 25.5 & $1.61(\mathrm{~s})$ \\
\hline 3 & 88.8 & $3.38(\mathrm{dd}, 4.5,11.5)$ & 27 & 25.1 & $1.62(\mathrm{~s})$ \\
\hline 4 & 39.7 & - & 28 & 28.2 & $1.33(\mathrm{~s})$ \\
\hline 5 & 56.4 & 0.74 (br. d, ca. 12) & 29 & 16.8 & $1.02(\mathrm{~s})$ \\
\hline 6 & 18.5 & $1.39,1.54$ (both m) & 30 & 17.2 & $0.93(\mathrm{~s})$ \\
\hline 7 & 35.1 & $1.22,1.50$ (both m) & $1^{\prime}$ & 107.1 & $4.98(\mathrm{~d}, 7.5)$ \\
\hline 8 & 40.1 & - & $2^{\prime}$ & 75.8 & $4.08(\mathrm{dd}, 7.5,8.5)$ \\
\hline 9 & 50.1 & $1.42(\mathrm{~m})$ & $3^{\prime}$ & 78.8 & $4.29(\mathrm{dd}, 8.5,9.0)$ \\
\hline 10 & 37.0 & - & $4^{\prime}$ & 71.9 & $4.26(\mathrm{dd}, 9.0,9.0)$ \\
\hline 11 & 30.9 & $\begin{array}{c}1.55(\mathrm{~m} \text {, overlapped }) \\
2.02(\mathrm{~m})\end{array}$ & $5^{\prime}$ & 78.5 & $4.06(\mathrm{~m}$, overlapped $)$ \\
\hline 12 & 70.4 & $4.08(\mathrm{~m})$ & $6^{\prime}$ & 63.1 & $\begin{array}{l}4.44 \text { (dd, } 5.5,11.5) \\
4.64 \text { (br. d, ca. 12) }\end{array}$ \\
\hline 13 & 49.7 & $2.04(\mathrm{dd}, 10.5,10.5)$ & $1^{\prime \prime}$ & 98.3 & $5.20(\mathrm{~d}, 7.5)$ \\
\hline 14 & 51.5 & - & $2^{\prime \prime}$ & 75.0 & $3.95(\mathrm{dd}, 7.5,8.5)$ \\
\hline 15 & 30.6 & $\begin{array}{c}0.99(\mathrm{~m}) \\
1.60(\mathrm{~m}, \text { overlapped })\end{array}$ & $3^{\prime \prime}$ & 79.0 & $4.21(\mathrm{~m}$, overlapped $)$ \\
\hline 16 & 26.4 & $1.47,1.80$ (both m) & $4^{\prime \prime}$ & 71.6 & $4.13(\mathrm{~m}$, overlapped $)$ \\
\hline 17 & 51.9 & 2.49 (q like, $c a .11)$ & $5^{\prime \prime}$ & 76.9 & $4.13(\mathrm{~m}$, overlapped $)$ \\
\hline 18 & 16.0 & $1.02(\mathrm{~s})$ & $6^{\prime \prime}$ & 69.9 & $\begin{array}{c}4.33(\mathrm{~m} \text {, overlapped) } \\
4.76(\mathrm{br} . \mathrm{d}, \mathrm{ca} .11)\end{array}$ \\
\hline 19 & 16.3 & $0.85(\mathrm{~s})$ & $1^{\prime \prime \prime}$ & 105.7 & $5.00(\mathrm{~d}, 7.5)$ \\
\hline 20 & 83.2 & - & $2^{\prime \prime \prime}$ & 74.9 & $4.05(\mathrm{dd}, 7.5,8.0)$ \\
\hline 21 & 23.3 & $1.62(\mathrm{~s})$ & $3^{\prime \prime \prime}$ & 78.1 & $4.15(\mathrm{dd}, 8.0,9.0)$ \\
\hline 22 & 39.7 & $\begin{array}{l}2.85(\mathrm{dd}, 8.0,13.5) \\
3.14(\mathrm{dd}, 5.5,13.5)\end{array}$ & $4^{\prime \prime \prime}$ & 71.1 & $4.22(\mathrm{~m})$ \\
\hline 23 & 126.8 & $6.21(\mathrm{ddd}, 5.5,8.0,16.0)$ & $5^{\prime \prime \prime}$ & 67.1 & $\begin{array}{c}3.74(\mathrm{dd}, 10.5,10.5) \\
4.34(\mathrm{~m}, \text { overlapped })\end{array}$ \\
\hline 24 & 138.1 & $6.13(d, 16.0)$ & & & \\
\hline
\end{tabular}

Table 3. The ${ }^{1} \mathrm{H}$ and ${ }^{13} \mathrm{C}-\mathrm{NMR}$ data for 3 in $\mathrm{C}_{5} \mathrm{D}_{5} \mathrm{~N}$.

\begin{tabular}{|c|c|c|c|c|c|}
\hline No. & $\delta_{\mathrm{C}}$ & $\delta_{\mathrm{H}}(J$ in $\mathrm{Hz})$ & No. & $\delta_{\mathrm{C}}$ & $\delta_{\mathrm{H}}(J$ in $\mathrm{Hz})$ \\
\hline 1 & 39.2 & $\begin{array}{c}0.76(\mathrm{~m}) \\
1.54(\mathrm{~m} \text {, overlapped })\end{array}$ & 30 & 17.2 & $0.88(\mathrm{~s})$ \\
\hline 2 & 26.8 & $1.81,2.15$ (both m) & $1^{\prime}$ & 104.8 & $4.92(\mathrm{~d}, 7.5)$ \\
\hline 3 & 89.0 & $3.28(\mathrm{dd}, 4.5,11.5)$ & $2^{\prime}$ & 82.9 & $4.09(\mathrm{~m}$, overlapped $)$ \\
\hline 4 & 39.9 & - & $3^{\prime}$ & 78.7 & $4.33(\mathrm{~m}$, overlapped $)$ \\
\hline 5 & 56.4 & 0.69 (br. d, ca. 12) & $4^{\prime}$ & 71.2 & $4.09(\mathrm{~m}$, overlapped $)$ \\
\hline 6 & 18.5 & $\begin{array}{c}1.46(\mathrm{~m} \text {, overlapped }) \\
1.53(\mathrm{~m})\end{array}$ & $5^{\prime}$ & 78.3 & $3.94(\mathrm{~m})$ \\
\hline 7 & 35.1 & $\begin{array}{c}1.20(\mathrm{~m}) \\
1.46(\mathrm{~m}, \text { overlapped })\end{array}$ & $6^{\prime}$ & 63.0 & $\begin{array}{c}4.35(\mathrm{~m} \text {, overlapped }) \\
4.56(\mathrm{dd}, 2.0,12.0)\end{array}$ \\
\hline 8 & 40.1 & $-\quad 11$ & $1^{\prime \prime}$ & 103.2 & $5.50(\mathrm{~d}, 7.5)$ \\
\hline 9 & 50.1 & $1.36(\mathrm{~m})$ & $2^{\prime \prime}$ & 84.6 & $4.18(\mathrm{~m}$, overlapped $)$ \\
\hline 10 & 36.9 & - & $3^{\prime \prime}$ & 77.9 & $4.28(\mathrm{dd}, 8.5,9.5)$ \\
\hline 11 & 30.8 & $\begin{array}{c}1.55(\mathrm{~m}, \text { overlapped }) \\
1.98(\mathrm{~m})\end{array}$ & $4^{\prime \prime}$ & 71.8 & $4.19(\mathrm{~m}$, overlapped $)$ \\
\hline 12 & 70.7 & $3.99(\mathrm{~m})$ & $5^{\prime \prime}$ & 77.7 & $3.85(\mathrm{~m})$ \\
\hline 13 & 49.5 & $2.02(\mathrm{dd}, 10.5,10.5)$ & $6^{\prime \prime}$ & 62.9 & $\begin{array}{c}4.35 \text { (m, overlapped }) \\
4.46(\mathrm{dd}, 2.5,11.5)\end{array}$ \\
\hline 14 & 51.5 & - & $1^{\prime \prime \prime}$ & 106.4 & $5.39(\mathrm{~d}, 7.0)$ \\
\hline 15 & 30.7 & $\begin{array}{c}0.97(\mathrm{~m}) \\
1.54(\mathrm{~m} \text {, overlapped })\end{array}$ & $2^{\prime \prime \prime}$ & 75.9 & $4.09(\mathrm{~m}$, overlapped $)$ \\
\hline 16 & 26.4 & $\begin{array}{c}1.46(\mathrm{~m} \text {, overlapped }) \\
1.79(\mathrm{~m})\end{array}$ & $3^{\prime \prime \prime}$ & 77.8 & $4.12(\mathrm{~m}$, overlapped $)$ \\
\hline 17 & 52.1 & 2.43 (q like, $c a .11)$ & $4^{\prime \prime \prime}$ & 70.7 & $4.12(\mathrm{~m}$, overlapped $)$ \\
\hline 18 & 16.0 & $1.01(\mathrm{~s})$ & $5^{\prime \prime \prime}$ & 67.4 & $\begin{array}{c}3.68(\mathrm{dd}, 10.0,11.0) \\
4.32(\mathrm{~m}, \text { overlapped })\end{array}$ \\
\hline 19 & 16.3 & $0.83(\mathrm{~s})$ & $1^{\prime \prime \prime \prime \prime}$ & 98.2 & $5.17(\mathrm{~d}, 7.5)$ \\
\hline 20 & 83.2 & - & $2^{\prime \prime \prime \prime}$ & 75.2 & $3.95(\mathrm{dd}, 7.5,9.0)$ \\
\hline 21 & 23.3 & $1.61(\mathrm{~s})$ & $3^{\prime \prime \prime \prime}$ & 78.9 & $4.18(\mathrm{dd}, 9.0,9.0)$ \\
\hline 22 & 39.8 & $\begin{array}{l}2.81(\mathrm{dd}, 8.0,14.5) \\
3.10(\mathrm{dd}, 6.0,14.5)\end{array}$ & $4^{\prime \prime \prime \prime}$ & 72.0 & $4.03(\mathrm{dd}, 9.0,9.0)$ \\
\hline 23 & 126.7 & $6.14(\mathrm{ddd}, 6.0,8.0,16.0)$ & $5^{\prime \prime \prime \prime}$ & 76.5 & $4.04(\mathrm{~m})$ \\
\hline 24 & 138.0 & $6.10(d, 16.0)$ & $6^{\prime \prime \prime \prime}$ & 68.4 & $\begin{array}{c}4.12 \text { (m, overlapped) } \\
4.65(\mathrm{dd}, 1.5,11.5)\end{array}$ \\
\hline 25 & 81.3 & - & $1^{\prime \prime \prime \prime \prime \prime}$ & 110.2 & $5.64(\mathrm{~d}, 1.5)$ \\
\hline 26 & 25.4 & $1.60(\mathrm{~s})$ & $2^{\prime \prime \prime \prime \prime}$ & 83.3 & $4.85(\mathrm{dd}, 1.5,3.0)$ \\
\hline 27 & 25.1 & $1.60(\mathrm{~s})$ & $3^{\prime \prime \prime \prime \prime}$ & 78.8 & $4.77(\mathrm{~m}$, overlapped $)$ \\
\hline 28 & 28.1 & $1.28(\mathrm{~s})$ & $4^{\prime \prime \prime \prime \prime}$ & 86.0 & 4.77 (m, overlapped) \\
\hline 29 & 16.7 & $1.11(\mathrm{~s})$ & $5^{\prime \prime \prime \prime \prime \prime}$ & 62.8 & $\begin{array}{c}4.19(\mathrm{~m} \text {, overlapped }) \\
4.29(\mathrm{dd}, 3.0,12.0)\end{array}$ \\
\hline
\end{tabular}


Notoginsenoside NL-A $\mathrm{A}_{4}(4)$ was a white powder. Its molecular formula was determined to be $\mathrm{C}_{44} \mathrm{H}_{72} \mathrm{O}_{17}$, on the basis of the ESI-Q-Orbitrap MS $\left(\mathrm{m} / z 871.46875[\mathrm{M}-\mathrm{H}]^{-}\right.$; calcd. for $\mathrm{C}_{44} \mathrm{H}_{71} \mathrm{O}_{17}$, 871.46858) and NMR data (Table 4) analysis. The IR spectrum showed absorption bands at 3379, 1718,1646 , and $1080 \mathrm{~cm}^{-1}$, corresponding to hydroxyl, carbonyl, olefin, and ether groups, respectively. By comparison, the ${ }^{1} \mathrm{H}$ and ${ }^{13} \mathrm{C}-\mathrm{NMR}$ spectra with those of $\mathbf{1}, \mathbf{2}$, and 3 , the aglycone of 4 was also proposed to be $3 \beta, 12 \beta, 20 S$-trihydroxy-25-hydroperoxydammar-23-ene. Its ${ }^{1} \mathrm{H}$ and ${ }^{13} \mathrm{C}$-NMR spectra displayed the signals that could be assigned to one $\beta$-D-glucopyranosyl $\left[\delta 5.20\left(1 \mathrm{H}, \mathrm{d}, J=7.0 \mathrm{~Hz}, \mathrm{H}-1^{\prime \prime}\right)\right]$ and one $\alpha$-L-arabinopyranosyl $\left[\delta 5.02\left(1 \mathrm{H}, \mathrm{d}, J=6.0 \mathrm{~Hz}, \mathrm{H}-1^{\prime \prime \prime}\right)\right]$. Forty-four signals were observed in its ${ }^{13} \mathrm{C}-\mathrm{NMR}$ spectrum. Except for the signals that belonged to the above-mentioned aglycone and glycosyl groups, the other three signals could be assigned to two ester carbonyls $\left[\delta_{C} 168.3\left(\mathrm{C}-1^{\prime}\right), 169.9\right.$ $\left.\left(\mathrm{C}-3^{\prime}\right)\right]$ together with one methylene $\left[\delta_{C} 43.8\left(\mathrm{C}-2^{\prime}\right)\right]$. Combining with the chemical shift $[\delta 3.83(2 \mathrm{H}$, $\left.\left.\mathrm{s}, \mathrm{H}_{2}-2^{\prime}\right)\right]$ of methylene proton, the presence of malonyl was deduced, which was confirmed by the cross peaks from $\delta_{\mathrm{H}} 3.83\left(\mathrm{H}_{2}-2^{\prime}\right)$ to $\delta_{\mathrm{C}} 168.3\left(\mathrm{C}-1^{\prime}\right), 169.9\left(\mathrm{C}-3^{\prime}\right)$. Moreover, the covalent connectivities of above-mentioned moieties were established by the long-range correlations from $\delta_{\mathrm{H}} 4.81(1 \mathrm{H}$, dd, $J=4.5,11.5 \mathrm{~Hz}, \mathrm{H}-3)$ to $\delta_{\mathrm{C}} 168.3\left(\mathrm{C}-1^{\prime}\right) ; \delta_{\mathrm{H}} 5.20\left(\mathrm{H}-1^{\prime \prime}\right)$ to $\delta_{\mathrm{C}} 83.3(\mathrm{C}-20) ; \delta_{\mathrm{H}} 5.02\left(\mathrm{H}-1^{\prime \prime \prime}\right)$ to $\delta_{\mathrm{C}} 69.0\left(\mathrm{C}-6^{\prime \prime}\right)$ (Figure 2). The NMR data for aglycone of 4 were remarkably reminiscent of those of compounds 1, 2, and 3, besides $\mathrm{C}-2-4$. The 3-OH group was established as $\beta$-oriented (equatorial) from the NOESY correlations beween $\delta_{\mathrm{H}} 4.01(\mathrm{H}-12)$ and $\delta_{\mathrm{H}} 1.38(\mathrm{H}-9) ; \delta_{\mathrm{H}} 0.78(\mathrm{H}-5)$ and $\delta_{\mathrm{H}} 1.38(\mathrm{H}-9), 4.81(\mathrm{H}-3)$ and vicinal coupling constants of $\mathrm{H}-3$ with $\mathrm{H}_{2}-2\left({ }^{3} \mathrm{~J}=4.5,11.5 \mathrm{~Hz}\right)$. Then, its aglycone was also confirmed to be $3 \beta, 12 \beta, 20 S$-trihydroxy-25-hydroperoxydammar-23-ene. On the basis of the above-mentioned evidence, the structure of notoginsenoside $\mathrm{NL}^{-\mathrm{A}_{4}}(4)$ was determined.

Table 4. The ${ }^{1} \mathrm{H}$ and ${ }^{13} \mathrm{C}-\mathrm{NMR}$ data for 4 in $\mathrm{C}_{5} \mathrm{D}_{5} \mathrm{~N}$.

\begin{tabular}{|c|c|c|c|c|c|}
\hline No. & $\delta_{C}$ & $\delta_{\mathrm{H}}(J$ in $\mathrm{Hz})$ & No. & $\delta_{C}$ & $\delta_{\mathrm{H}}(J$ in $\mathrm{Hz})$ \\
\hline 1 & 38.3 & $\begin{array}{l}0.81 \text { (m, overlapped }) \\
1.59(\mathrm{~m}, \text { overlapped })\end{array}$ & 23 & 126.6 & $6.15(\mathrm{~m})$ \\
\hline 2 & 24.0 & $1.70,1.76$ (both m) & 24 & 138.1 & $6.15(\mathrm{~m})$ \\
\hline 3 & 81.3 & $4.81(\mathrm{dd}, 4.5,11.5)$ & 25 & 81.3 & - \\
\hline 4 & 38.6 & - & 26 & 25.4 & $1.62(\mathrm{~s})$ \\
\hline 5 & 56.1 & 0.78 (br. d, ca. 11) & 27 & 25.1 & $1.61(\mathrm{~s})$ \\
\hline 6 & 18.4 & $\begin{array}{l}1.38(\mathrm{~m}, \text { overlapped }) \\
1.47(\mathrm{~m}, \text { overlapped })\end{array}$ & 28 & 28.1 & $1.05(\mathrm{~s})$ \\
\hline 7 & 34.9 & $\begin{array}{c}1.19(\mathrm{~m}) \\
1.46(\mathrm{~m}, \text { overlapped })\end{array}$ & 29 & 16.8 & $0.94(\mathrm{~s})$ \\
\hline 8 & 40.0 & - & 30 & 17.2 & $0.87(\mathrm{~s})$ \\
\hline 9 & 50.1 & 1.38 (m, overlapped) & $1^{\prime}$ & 168.3 & - \\
\hline 10 & 37.1 & - & $2^{\prime}$ & 43.8 & $3.83(\mathrm{~s})$ \\
\hline 11 & 30.8 & $\begin{array}{c}1.58(\mathrm{~m}, \text { overlapped }) \\
1.99(\mathrm{~m})\end{array}$ & $3^{\prime}$ & 169.9 & - \\
\hline 12 & 70.4 & $4.01(\mathrm{~m})$ & $1^{\prime \prime}$ & 98.3 & $5.20(\mathrm{~d}, 7.0)$ \\
\hline 13 & 49.5 & $2.03(\mathrm{dd}, 10.0,10.0)$ & $2^{\prime \prime}$ & 75.1 & $3.96(\mathrm{dd}, 7.0,8.0)$ \\
\hline 14 & 51.4 & - & $3^{\prime \prime}$ & 78.8 & $4.21(\mathrm{dd}, 8.0,8.5)$ \\
\hline 15 & 30.5 & $\begin{array}{l}0.98 \text { (m, overlapped) } \\
1.58(\mathrm{~m}, \text { overlapped })\end{array}$ & $4^{\prime \prime}$ & 71.7 & $4.14(\mathrm{dd}, 8.5,9.0)$ \\
\hline 16 & 26.4 & $1.49,1.80$ (both m) & $5^{\prime \prime}$ & 76.7 & $4.10(\mathrm{~m})$ \\
\hline 17 & 52.0 & 2.45 (q like, ca. 10) & $6^{\prime \prime}$ & 69.0 & $\begin{array}{l}4.28(\mathrm{dd}, 4.0,10.5) \\
4.71(\text { br. d, ca. } 11)\end{array}$ \\
\hline 18 & 16.0 & $1.02(\mathrm{~s})$ & $1^{\prime \prime \prime}$ & 104.4 & $5.02(\mathrm{~d}, 6.0)$ \\
\hline 19 & 16.2 & $0.84(\mathrm{~s})$ & $2^{\prime \prime \prime}$ & 72.1 & $4.47(\mathrm{dd}, 6.0,6.5)$ \\
\hline 20 & 83.3 & - & $3^{\prime \prime \prime}$ & 74.1 & $4.25(\mathrm{~m})$ \\
\hline 21 & 23.3 & $1.62(\mathrm{~s})$ & $4^{\prime \prime \prime}$ & 68.5 & $4.38(\mathrm{~m})$ \\
\hline 22 & 40.1 & $\begin{array}{l}2.88(\mathrm{dd}, 6.5,13.5) \\
3.10(\mathrm{dd}, 4.0,13.5)\end{array}$ & $5^{\prime \prime \prime}$ & 65.4 & $\begin{array}{l}3.85 \text { (br. d, ca. 12) } \\
4.34 \text { (dd, 2.5, 12.0) }\end{array}$ \\
\hline
\end{tabular}

Notoginsenoside NL-A 4 (4) was a white powder. Its molecular formula was determined to be $\mathrm{C}_{44} \mathrm{H}_{72} \mathrm{O}_{17}$, on the basis of the ESI-Q-Orbitrap MS $\left(\mathrm{m} / z 871.46875[\mathrm{M}-\mathrm{H}]^{-}\right.$; calcd. for $\mathrm{C}_{44} \mathrm{H}_{71} \mathrm{O}_{17}$, 871.46858) and NMR data (Table 4) analysis. The IR spectrum showed absorption bands at 3379, 1718,1646 , and $1080 \mathrm{~cm}^{-1}$, corresponding to hydroxyl, carbonyl, olefin, and ether groups, respectively. By comparison, the ${ }^{1} \mathrm{H}$ and ${ }^{13} \mathrm{C}-\mathrm{NMR}$ spectra with those of 1,2 , and 3 , the aglycone of 4 was also proposed to be $3 \beta, 12 \beta, 20 S$-trihydroxy-25-hydroperoxydammar-23-ene. Its ${ }^{1} \mathrm{H}$ and ${ }^{13} \mathrm{C}-\mathrm{NMR}$ spectra 
displayed the signals that could be assigned to one $\beta$-D-glucopyranosyl $\left[\delta 5.20\left(1 \mathrm{H}, \mathrm{d}, J=7.0 \mathrm{~Hz}, \mathrm{H}-1^{\prime \prime}\right)\right]$ and one $\alpha$-L-arabinopyranosyl $\left[\delta 5.02\left(1 \mathrm{H}, \mathrm{d}, J=6.0 \mathrm{~Hz}, \mathrm{H}-1^{\prime \prime \prime}\right)\right]$. Forty-four signals were observed in its ${ }^{13} \mathrm{C}$-NMR spectrum. Except for the signals that belonged to the above-mentioned aglycone and glycosyl groups, the other three signals could be assigned to two ester carbonyls $\left[\delta_{\mathrm{C}} 168.3\left(\mathrm{C}-1^{\prime}\right), 169.9\right.$ $\left.\left(\mathrm{C}-3^{\prime}\right)\right]$ together with one methylene $\left[\delta_{\mathrm{C}} 43.8\left(\mathrm{C}-2^{\prime}\right)\right]$. Combining with the chemical shift $[\delta 3.83(2 \mathrm{H}$, $\left.\mathrm{s}, \mathrm{H}_{2}-2^{\prime}\right)$ ] of methylene proton, the presence of malonyl was deduced, which was confirmed by the cross peaks from $\delta_{\mathrm{H}} 3.83\left(\mathrm{H}_{2}-2^{\prime}\right)$ to $\delta_{\mathrm{C}} 168.3\left(\mathrm{C}-1^{\prime}\right), 169.9\left(\mathrm{C}-3^{\prime}\right)$. Moreover, the covalent connectivities of above-mentioned moieties were established by the long-range correlations from $\delta_{\mathrm{H}} 4.81(1 \mathrm{H}, \mathrm{dd}$, $J=4.5,11.5 \mathrm{~Hz}, \mathrm{H}-3)$ to $\delta_{\mathrm{C}} 168.3\left(\mathrm{C}-1^{\prime}\right) ; \delta_{\mathrm{H}} 5.20\left(\mathrm{H}-1^{\prime \prime}\right)$ to $\delta_{\mathrm{C}} 83.3(\mathrm{C}-20) ; \delta_{\mathrm{H}} 5.02\left(\mathrm{H}-1^{\prime \prime \prime}\right)$ to $\delta_{\mathrm{C}} 69.0\left(\mathrm{C}-6^{\prime \prime}\right)$ (Figure 2). The NMR data for aglycone of 4 were remarkably reminiscent of those of compounds 1, 2, and 3, besides C-2-4. The 3-OH group was established as $\beta$-oriented (equatorial) from the NOESY correlations beween $\delta_{\mathrm{H}} 4.01(\mathrm{H}-12)$ and $\delta_{\mathrm{H}} 1.38(\mathrm{H}-9) ; \delta_{\mathrm{H}} 0.78(\mathrm{H}-5)$ and $\delta_{\mathrm{H}} 1.38(\mathrm{H}-9), 4.81(\mathrm{H}-3)$ and vicinal coupling constants of $\mathrm{H}-3$ with $\mathrm{H}_{2}-2\left({ }^{3} \mathrm{~J}=4.5,11.5 \mathrm{~Hz}\right)$. Then, its aglycone was also confirmed to be $3 \beta, 12 \beta, 20 S$-trihydroxy-25-hydroperoxydammar-23-ene. On the basis of the above-mentioned evidence, the structure of notoginsenoside NL-A 4 (4) was determined.

Notoginsenoside NL-B ${ }_{1}(5)$ was obtained as a white powder with positive optical rotation $\left([\alpha]_{\mathrm{D}}^{25}\right.$ +2.2, MeOH). Its IR spectrum exhibited absorption maxima at 3379, 1649 and $1076 \mathrm{~cm}^{-1}$, accounting for the hydroxyl, olefinic bond, and ether function, respectively. D-glucose and L-arabinose were detected from its acid hydrolysis product [12]. Its ${ }^{1} \mathrm{H}$ and ${ }^{13} \mathrm{C}-\mathrm{NMR}$ (Table 5) spectra revealed the difference only in H-23-27 [ $\delta 1.56,1.57$ (3H each, both s, $\mathrm{H}_{3}-26$, and 27), $6.09(1 \mathrm{H}, \mathrm{d}, J=15.5 \mathrm{~Hz}, \mathrm{H}-24), 6.23(1 \mathrm{H}$, ddd, $J=5.5,8.5,15.5 \mathrm{~Hz}, \mathrm{H}-23)]$ and C-23-27 [ $\delta_{\mathrm{C}} 30.6(\mathrm{C}-27), 30.7$ (C-26), 70.0 (C-25), 122.7 (C-23), 142.2 (C-24)], as compared to that of compound 1. The ESI-Q-Orbitrap MS analysis result indicated its molecular formula was $\mathrm{C}_{47} \mathrm{H}_{80} \mathrm{O}_{18}\left(\mathrm{~m} / z\right.$ 931.52838 $[\mathrm{M}-\mathrm{H}]^{-}$; calcd. for $\mathrm{C}_{47} \mathrm{H}_{79} \mathrm{O}_{18}$, 931.52609), an oxygen atom disappeared when comparing it with 1, suggesting that C-25 of 5 was substituted by the hydroxyl group. The cross peaks from $\delta_{\mathrm{H}} 1.57\left(\mathrm{H}_{3}-26\right)$ to $\delta_{\mathrm{C}} 30.6(\mathrm{C}-27), 70.0(\mathrm{C}-25), 142.2$ $(\mathrm{C}-24) ; \delta_{\mathrm{H}} 1.56\left(\mathrm{H}_{3}-27\right)$ to $\delta_{\mathrm{C}} 30.7$ (C-26), 70.0 (C-25), 142.2 (C-24), clarified the above-mentioned description. The NMR data of its aglycone were likable to those of the known compound, 3-O- $\beta$-D-xylopyranosyl-( $1 \rightarrow 2)-\beta$-D-glucopyranosyl- $(1 \rightarrow 2)-\beta$-D-glucopyranosyl-3 $\beta, 12 \beta, 20(S), 25$ -tetrahydroxydammar-23-ene-20-O- $\beta$-D-glucopyranoside- $(1 \rightarrow 6)-\beta$-D-glucopyranoside [6]. Thus, its aglycone was elucidated to be $3 \beta, 12 \beta, 20(S), 25$-tetrahydroxydammar-23-ene. Finally, the linkage positions between sugar and sugar, as well as sugar and aglycone were clarified by the long-range correlation observations from $\delta_{\mathrm{H}} 4.96\left(\mathrm{H}-1^{\prime}\right)$ to $\delta_{\mathrm{C}} 89.0(\mathrm{C}-3) ; \delta_{\mathrm{H}} 5.18\left(\mathrm{H}-1^{\prime \prime}\right)$ to $\delta_{\mathrm{C}} 83.3(\mathrm{C}-20)$; and $\delta_{\mathrm{H}}$ $5.65\left(\mathrm{H}-1^{\prime \prime \prime}\right)$ to $\delta_{\mathrm{C}} 68.4\left(\mathrm{C}-6^{\prime \prime}\right)$.

The molecular formula of both notoginsenosides $\mathrm{NL}_{2} \mathrm{~B}_{2}(6)$ and NL-B 3 (7) were determined to be $\mathrm{C}_{58} \mathrm{H}_{98} \mathrm{O}_{27}$, on the basis of the MS analysis (6: $\mathrm{m} / \mathrm{z} 1225.62134[\mathrm{M}-\mathrm{H}]^{-}, 7: \mathrm{m} / z$ 1225. 61841 [M $-\mathrm{H}]^{-}$, both calcd. for $\mathrm{C}_{58} \mathrm{H}_{97} \mathrm{O}_{27}, 1225.62117$ ). Based on their ${ }^{1} \mathrm{H}$ - and ${ }^{13} \mathrm{C}-\mathrm{NMR}$ (Tables 6 and 7 ), ${ }^{1} \mathrm{H}-{ }^{1} \mathrm{H}$ COSY, HSQC, HMBC, and HSQC-TOCSY spectral characteristics, the aglycones of both 6 and 7 were identified as $3 \beta, 12 \beta, 20(S), 25$-tetrahydroxydammar-23-ene, too. Moreover, by comparing to 3 , we could deduce that 6 had the same glycosyl moieties as 3. D-glucose, L-arabinose, and D-xylose were yielded from its acid hydrolysis reaction [12]. Moreover, the long-range correlations from $\delta_{\mathrm{H}} 4.92$ $\left(\mathrm{H}-1^{\prime}\right)$ to $\delta_{\mathrm{C}} 89.0(\mathrm{C}-3) ; \delta_{\mathrm{H}} 5.50\left(\mathrm{H}-1^{\prime \prime}\right)$ to $\delta_{\mathrm{C}} 83.0\left(\mathrm{C}-2^{\prime}\right) ; \delta_{\mathrm{H}} 5.39\left(\mathrm{H}-1^{\prime \prime \prime}\right)$ to $\delta_{\mathrm{C}} 84.6\left(\mathrm{C}-2^{\prime \prime}\right) ; \delta_{\mathrm{H}} 5.16\left(\mathrm{H}-1^{\prime \prime \prime \prime}\right)$ to $\delta_{\mathrm{C}} 83.4(\mathrm{C}-20)$; and $\delta_{\mathrm{H}} 5.64\left(\mathrm{H}-1^{\prime \prime \prime \prime \prime}\right)$ to $\delta_{\mathrm{C}} 68.4\left(\mathrm{C}-6^{\prime \prime \prime \prime}\right)$ were observed in the HMBC experiment (Figure 2). Those results confirmed that the glycosyl moieties of 6 were matched with 3. Meanwhile, only $\mathrm{D}$-glucose and $\mathrm{D}$-xylose were detected from the acid hydrolysis product of 7 . In addition, the cross peaks displayed in its HMBC spectrum (Figure 2) denoted that the C-20-substituted glycosyl in 7 was $\beta$-D-xylopyranosyl $(1 \rightarrow 6)-\beta$-D-glucopyranosyl $\left[\delta 4.98\left(1 \mathrm{H}, \mathrm{d}, J=7.2 \mathrm{~Hz}, \mathrm{H}-1^{\prime \prime \prime \prime \prime}\right), 5.18(1 \mathrm{H}, \mathrm{d}, J=7.8 \mathrm{~Hz}\right.$,

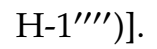


Table 5. The ${ }^{1} \mathrm{H}$ and ${ }^{13} \mathrm{C}-\mathrm{NMR}$ data for 5 in $\mathrm{C}_{5} \mathrm{D}_{5} \mathrm{~N}$.

\begin{tabular}{|c|c|c|c|c|c|}
\hline No. & $\delta_{C}$ & $\delta_{H}(J$ in $\mathrm{Hz})$ & No. & $\delta_{C}$ & $\delta_{H}(J$ in $\mathrm{Hz})$ \\
\hline 1 & 39.1 & $\begin{array}{c}0.78(\mathrm{~m}) \\
1.55(\mathrm{~m}, \text { overlapped })\end{array}$ & 25 & 70.0 & - \\
\hline 2 & 26.8 & $\begin{array}{c}1.79(\mathrm{~m} \text {, overlapped }) \\
2.22(\mathrm{~m})\end{array}$ & 26 & 30.7 & $1.56(\mathrm{~s})$ \\
\hline 3 & 89.0 & $3.37(\mathrm{dd}, 4.5,12.0)$ & 27 & 30.6 & $1.57(\mathrm{~s})$ \\
\hline 4 & 39.7 & - & 28 & 28.1 & $1.28(\mathrm{~s})$ \\
\hline 5 & 56.4 & 0.73 (br. d, ca. 12) & 29 & 16.7 & $1.11(\mathrm{~s})$ \\
\hline 6 & 18.4 & $\begin{array}{c}1.38(\mathrm{~m} \text {, overlapped }) \\
1.50(\mathrm{~m})\end{array}$ & 30 & 17.2 & $0.89(\mathrm{~s})$ \\
\hline 7 & 35.1 & $\begin{array}{c}1.20(\mathrm{~m}) \\
1.48(\mathrm{~m}, \text { overlapped })\end{array}$ & $1^{\prime}$ & 107.0 & $4.96(\mathrm{~d}, 8.0)$ \\
\hline 8 & 40.1 & - & $2^{\prime}$ & 75.8 & $4.06(\mathrm{dd}, 8.0,9.0)$ \\
\hline 9 & 50.1 & 1.37 (m, overlapped) & $3^{\prime}$ & 78.8 & $4.28(\mathrm{dd}, 9.0,9.0)$ \\
\hline 10 & 36.9 & - & $4^{\prime}$ & 71.8 & $4.24(\mathrm{dd}, 9.0,9.0)$ \\
\hline 11 & 30.8 & $\begin{array}{c}1.56(\mathrm{~m}, \text { overlapped }) \\
2.04(\mathrm{~m})\end{array}$ & $5^{\prime}$ & 78.4 & $4.05(\mathrm{~m}$, overlapped $)$ \\
\hline 12 & 70.6 & $4.04(\mathrm{~m})$ & $6^{\prime}$ & 63.1 & $\begin{array}{c}4.44(\mathrm{dd}, J=5.5,11.5) \\
4.63(\mathrm{dd}, 1.5,11.5)\end{array}$ \\
\hline 13 & 49.5 & $2.04(\mathrm{dd}, 11.0,11.0)$ & $1^{\prime \prime}$ & 98.2 & $5.18(\mathrm{~d}, 7.5)$ \\
\hline 14 & 51.5 & - & $2^{\prime \prime}$ & 75.2 & 3.98 (dd, $7.5,8.5)$ \\
\hline 15 & 30.5 & $\begin{array}{c}0.96(\mathrm{~m}) \\
1.56(\mathrm{~m}, \text { overlapped })\end{array}$ & $3^{\prime \prime}$ & 78.9 & $4.18(\mathrm{dd}, 8.5,8.5)$ \\
\hline 16 & 26.4 & $1.47,1.79$ (both m, overlapped) & $4^{\prime \prime}$ & 72.0 & $4.01(\mathrm{dd}, 8.5,9.0)$ \\
\hline 17 & 52.1 & 2.44 (q like, ca. 10) & $5^{\prime \prime}$ & 76.6 & $4.09(\mathrm{~m})$ \\
\hline 18 & 16.0 & $1.01(\mathrm{~s})$ & $6^{\prime \prime}$ & 68.4 & $\begin{array}{l}4.21(\mathrm{dd}, 3.0,11.0) \\
4.69 \text { (br. d, ca. 11) }\end{array}$ \\
\hline 19 & 16.3 & $0.83(\mathrm{~s})$ & $1^{\prime \prime \prime}$ & 110.2 & $5.65(\mathrm{~d}, 1.5)$ \\
\hline 20 & 83.3 & - & $2^{\prime \prime \prime}$ & 83.5 & 4.89 (br. s) \\
\hline 21 & 23.3 & $1.61(\mathrm{~s})$ & $3^{\prime \prime \prime}$ & 78.9 & $4.80(\mathrm{~m}$, overlapped $)$ \\
\hline 22 & 39.6 & $\begin{array}{l}2.84(\mathrm{dd}, 8.5,14.0) \\
3.11(\mathrm{dd}, 5.5,14.0)\end{array}$ & $4^{\prime \prime \prime}$ & 86.0 & $4.80(\mathrm{~m}$, overlapped $)$ \\
\hline 23 & 122.7 & $6.23(\mathrm{ddd}, 5.5,8.5,15.5)$ & $5^{\prime \prime \prime}$ & 62.8 & $\begin{array}{l}4.21(\mathrm{dd}, 3.5,12.0) \\
4.32 \text { (br. d, ca. 12) }\end{array}$ \\
\hline 24 & 142.2 & $6.09(\mathrm{~d}, 15.5)$ & & & \\
\hline
\end{tabular}

Table 6. The ${ }^{1} \mathrm{H}$ and ${ }^{13} \mathrm{C}-\mathrm{NMR}$ data for 6 in $\mathrm{C}_{5} \mathrm{D}_{5} \mathrm{~N}$.

\begin{tabular}{|c|c|c|c|c|c|}
\hline No. & $\delta_{\mathrm{C}}$ & $\delta_{\mathrm{H}}(J$ in $\mathrm{Hz})$ & No. & $\delta_{\mathrm{C}}$ & $\delta_{\mathrm{H}}(J$ in $\mathrm{Hz})$ \\
\hline 1 & 39.2 & $0.76,1.52$ (both m) & 30 & 17.2 & $0.89(\mathrm{~s})$ \\
\hline 2 & 26.8 & $1.81,2.18$ (both m) & $1^{\prime}$ & 104.8 & $4.92(\mathrm{~d}, 8.0)$ \\
\hline 3 & 89.0 & $3.28(\mathrm{dd}, 4.0,11.5)$ & $2^{\prime}$ & 83.0 & $4.11(\mathrm{dd}, 7.0,8.0)$ \\
\hline 4 & 39.8 & - & $3^{\prime}$ & 78.7 & 4.35 (m, overlapped) \\
\hline 5 & 56.4 & 0.69 (br. d, ca. 12) & $4^{\prime}$ & 71.2 & $4.08(\mathrm{dd}, 9.0,9.0)$ \\
\hline 6 & 18.5 & $\begin{array}{c}1.37(\mathrm{~m}, \text { overlapped }) \\
1.49(\mathrm{~m})\end{array}$ & $5^{\prime}$ & 78.3 & $3.94(\mathrm{~m})$ \\
\hline 7 & 35.1 & $1.20,1.45$ (both m) & $6^{\prime}$ & 63.0 & $\begin{array}{c}4.35(\mathrm{~m} \text {, overlapped }) \\
4.57(\mathrm{dd}, 2.0,11.5)\end{array}$ \\
\hline 8 & 40.1 & - & $1^{\prime \prime}$ & 103.2 & $5.50(\mathrm{~d}, 7.5)$ \\
\hline 9 & 50.1 & 1.37 (m, overlapped) & $2^{\prime \prime}$ & 84.6 & $4.19(\mathrm{~m}$, overlapped $)$ \\
\hline 10 & 36.9 & - & $3^{\prime \prime}$ & 78.0 & $4.27(\mathrm{dd}, J=9.0,9.0)$ \\
\hline 11 & 30.8 & $\begin{array}{c}1.55(\mathrm{~m} \text {, overlapped }) \\
1.98(\mathrm{~m})\end{array}$ & $4^{\prime \prime}$ & 71.9 & $4.20(\mathrm{dd}, 7.0,9.0)$ \\
\hline 12 & 70.6 & $3.99(\mathrm{~m}$, overlapped $)$ & $5^{\prime \prime}$ & 77.8 & $3.85(\mathrm{~m})$ \\
\hline 13 & 49.5 & $2.04(\mathrm{dd}, 10.5,10.5)$ & $6^{\prime \prime}$ & 62.9 & $\begin{array}{c}4.35(\mathrm{~m} \text {, overlapped }) \\
4.46(\mathrm{dd}, 3.0,11.5)\end{array}$ \\
\hline 14 & 51.5 & - & $1^{\prime \prime \prime}$ & 106.5 & $5.39(\mathrm{~d}, 6.5)$ \\
\hline 15 & 30.6 & $\begin{array}{c}0.96(\mathrm{~m}) \\
1.55(\mathrm{~m} \text {, overlapped })\end{array}$ & $2^{\prime \prime \prime}$ & 76.0 & $4.10(\mathrm{dd}, 6.5,9.0)$ \\
\hline 16 & 26.4 & $1.47,1.77$ (both m) & $3^{\prime \prime \prime}$ & 77.7 & 4.12 (m, overlapped) \\
\hline 17 & 52.1 & 2.44 (q like, $c a .11)$ & $4^{\prime \prime \prime}$ & 70.7 & $4.13(\mathrm{dd}, 9.0,9.0)$ \\
\hline 18 & 16.0 & $1.01(\mathrm{~s})$ & $5^{\prime \prime \prime}$ & 67.4 & $\begin{array}{c}3.68(\mathrm{dd}, 11.0,11.0) \\
4.30(\mathrm{~m} \text {, overlapped })\end{array}$ \\
\hline 19 & 16.3 & $0.83(\mathrm{~s})$ & $1^{\prime \prime \prime \prime \prime}$ & 98.2 & $5.16(\mathrm{~d}, 7.5)$ \\
\hline 20 & 83.4 & - & $2^{\prime \prime \prime \prime}$ & 75.2 & $3.95(\mathrm{dd}, 7.5,8.0)$ \\
\hline 21 & 23.3 & $1.60(\mathrm{~s})$ & $3^{\prime \prime \prime \prime}$ & 78.9 & $4.16(\mathrm{dd}, 8.0,9.5)$ \\
\hline 22 & 39.6 & $\begin{array}{l}2.82(\mathrm{dd}, 8.5,14.0) \\
3.09(\mathrm{dd}, 6.0,14.0)\end{array}$ & $4^{\prime \prime \prime \prime}$ & 72.0 & $3.98(\mathrm{dd}, 9.0,9.5)$ \\
\hline 23 & 122.8 & $6.21(\mathrm{ddd}, 6.0,8.5,15.5)$ & $5^{\prime \prime \prime \prime}$ & 76.5 & $4.06(\mathrm{~m})$ \\
\hline 24 & 142.3 & $6.07(\mathrm{~d}, 15.5)$ & $6^{\prime \prime \prime \prime}$ & 68.4 & $\begin{array}{c}4.07 \text { (m, overlapped) } \\
4.65 \text { (br. d, ca. 11) }\end{array}$ \\
\hline 25 & 70.0 & - & $1^{\prime \prime \prime \prime \prime \prime}$ & 110.2 & $5.64(\mathrm{~d}, 1.5)$ \\
\hline 26 & 30.7 & $1.55(\mathrm{~s})$ & $2^{\prime \prime \prime \prime \prime \prime}$ & 83.3 & $4.85(\mathrm{dd}, 1.5,3.0)$ \\
\hline 27 & 30.7 & $1.55(\mathrm{~s})$ & $3^{\prime \prime \prime \prime \prime \prime}$ & 79.0 & 4.77 (m, overlapped) \\
\hline 28 & 28.1 & $1.28(\mathrm{~s})$ & $4^{\prime \prime \prime \prime \prime}$ & 86.1 & 4.77 (m, overlapped) \\
\hline 29 & 16.7 & $1.11(\mathrm{~s})$ & $5^{\prime \prime \prime \prime \prime \prime}$ & 62.8 & $\begin{array}{l}4.19(\mathrm{~m} \text {, overlapped }) \\
4.30(\mathrm{~m}, \text { overlapped })\end{array}$ \\
\hline
\end{tabular}


Table 7. The ${ }^{1} \mathrm{H}$ and ${ }^{13} \mathrm{C}-\mathrm{NMR}$ data for 7 in $\mathrm{C}_{5} \mathrm{D}_{5} \mathrm{~N}$

\begin{tabular}{|c|c|c|c|c|c|}
\hline No. & $\delta_{C}$ & $\delta_{\mathrm{H}}(J$ in $\mathrm{Hz})$ & No. & $\delta_{C}$ & $\delta_{\mathrm{H}}(J$ in $\mathrm{Hz})$ \\
\hline 1 & 39.2 & $0.76,1.53$ (both m) & 30 & 17.2 & $0.91(\mathrm{~s})$ \\
\hline 2 & 26.8 & $1.80,2.19$ (both m) & $1^{\prime}$ & 104.8 & $4.94(\mathrm{~d}, 7.2)$ \\
\hline 3 & 89.0 & $3.30(\mathrm{dd}, 4.2,11.4)$ & $2^{\prime}$ & 82.8 & 4.14 (m, overlapped) \\
\hline 4 & 39.8 & - & $3^{\prime}$ & 78.7 & 4.37 (m, overlapped) \\
\hline 5 & 56.4 & 0.69 (br. d, ca. 11) & $4^{\prime}$ & 71.1 & $4.10(\mathrm{~m}$, overlapped $)$ \\
\hline 6 & 18.5 & $\begin{array}{c}1.37(\mathrm{~m} \text {, overlapped }) \\
1.47(\mathrm{~m})\end{array}$ & $5^{\prime}$ & 78.3 & $3.98(\mathrm{~m})$ \\
\hline 7 & 35.1 & $\begin{array}{c}1.21(\mathrm{~m}) \\
1.47(\mathrm{~m}, \text { overlapped })\end{array}$ & $6^{\prime}$ & 63.0 & $\begin{array}{c}4.37(\mathrm{~m} \text {, overlapped }) \\
4.59(\mathrm{dd}, 1.8,11.4)\end{array}$ \\
\hline 8 & 40.0 & - & $1^{\prime \prime}$ & 103.1 & $5.54(\mathrm{~d}, 7.8)$ \\
\hline 9 & 50.1 & 1.37 (m, overlapped) & $2^{\prime \prime}$ & 84.6 & $4.21(\mathrm{dd}, 7.8,9.0)$ \\
\hline 10 & 36.9 & - & $3^{\prime \prime}$ & 78.0 & $4.30(\mathrm{dd}, 9.0,9.0)$ \\
\hline 11 & 30.7 & $\begin{array}{c}1.55(\mathrm{~m} \text {, overlapped }) \\
1.99(\mathrm{~m})\end{array}$ & $4^{\prime \prime}$ & 71.8 & $4.22(\mathrm{~m}$, overlapped $)$ \\
\hline 12 & 70.5 & $4.05(\mathrm{~m})$ & $5^{\prime \prime}$ & 77.8 & $3.88(\mathrm{~m})$ \\
\hline 13 & 49.6 & $2.05(\mathrm{dd}, 10.8,10.8)$ & $6^{\prime \prime}$ & 62.9 & $\begin{array}{c}4.37 \text { (m, overlapped) } \\
4.51(\mathrm{dd}, 2.4,11.4)\end{array}$ \\
\hline 14 & 51.5 & - & $1^{\prime \prime \prime}$ & 106.5 & $5.41(\mathrm{~d}, 7.2)$ \\
\hline 15 & 30.6 & $\begin{array}{c}0.97(\mathrm{~m}) \\
1.55(\mathrm{~m}, \text { overlapped })\end{array}$ & $2^{\prime \prime \prime}$ & 76.0 & $4.12(\mathrm{dd}, 7.2,9.6)$ \\
\hline 16 & 26.4 & $\begin{array}{c}1.49(\mathrm{~m} \text {, overlapped }) \\
1.77(\mathrm{~m})\end{array}$ & $3^{\prime \prime \prime}$ & 77.9 & $4.15(\mathrm{~m}$, overlapped $)$ \\
\hline 17 & 52.0 & 2.47 (q like, $c a .10$ ) & $4^{\prime \prime \prime}$ & 70.7 & $4.16(\mathrm{~m}$, overlapped $)$ \\
\hline 18 & 16.0 & $1.00(\mathrm{~s})$ & $5^{\prime \prime \prime}$ & 67.4 & $\begin{array}{c}3.71(\mathrm{dd}, 11.4,11.4) \\
4.36(\mathrm{~m}, \text { overlapped })\end{array}$ \\
\hline 19 & 16.3 & $0.83(\mathrm{~s})$ & $1^{\prime \prime \prime \prime}$ & 98.2 & $5.18(\mathrm{~d}, 7.8)$ \\
\hline 20 & 83.3 & - & $2^{\prime \prime \prime \prime}$ & 75.1 & $3.95(\mathrm{dd}, 7.8,8.4)$ \\
\hline 21 & 23.2 & $1.61(\mathrm{~s})$ & $3^{\prime \prime \prime \prime}$ & 78.9 & $4.19(\mathrm{dd}, 8.4,9.0)$ \\
\hline 22 & 39.8 & $\begin{array}{l}2.86(\mathrm{dd}, 8.4,13.8) \\
3.13(\mathrm{dd}, 6.0,13.8)\end{array}$ & $4^{\prime \prime \prime \prime}$ & 71.6 & $4.10(\mathrm{~m}$, overlapped $)$ \\
\hline 23 & 122.8 & $6.24(\mathrm{ddd}, 6.0,8.4,15.6)$ & $5^{\prime \prime \prime \prime}$ & 76.9 & $4.10(\mathrm{~m}$, overlapped $)$ \\
\hline 24 & 142.3 & $6.10(\mathrm{~d}, 15.6)$ & $6^{\prime \prime \prime \prime}$ & 70.0 & $\begin{array}{l}4.28(\mathrm{dd}, 4.2,13.2) \\
4.76(\mathrm{dd}, 1.2,13.2)\end{array}$ \\
\hline 25 & 70.1 & - & $1^{\prime \prime \prime \prime \prime \prime}$ & 105.6 & $4.98(\mathrm{~d}, 7.2)$ \\
\hline 26 & 30.7 & $1.57(\mathrm{~s})$ & $2^{\prime \prime \prime \prime \prime \prime}$ & 74.9 & $4.04(\mathrm{dd}, 7.2,8.4)$ \\
\hline 27 & 30.6 & $1.56(\mathrm{~s})$ & $3^{\prime \prime \prime \prime \prime \prime}$ & 78.0 & 4.20 (m, overlapped) \\
\hline 28 & 28.1 & $1.29(\mathrm{~s})$ & $4^{\prime \prime \prime \prime \prime \prime}$ & 71.1 & $4.17(\mathrm{~m})$ \\
\hline 29 & 16.7 & $1.12(\mathrm{~s})$ & $5^{\prime \prime \prime \prime \prime \prime}$ & 67.0 & $\begin{array}{l}3.70(\mathrm{dd}, 9.6,9.6) \\
4.33(\mathrm{dd}, 4.8,9.6)\end{array}$ \\
\hline
\end{tabular}

Notoginsenoside NL- $\mathrm{C}_{1}$ (8), white powder, exhibited a molecular ion peak $[\mathrm{M}-\mathrm{H}]^{-}$at $\mathrm{m} / \mathrm{z}$ 929.51184 (calcd. for $\mathrm{C}_{47} \mathrm{H}_{77} \mathrm{O}_{18}, 929.51044$ ) in ESI-Q-Orbitrap MS spectrum, which matched to the molecular formula, $\mathrm{C}_{47} \mathrm{H}_{78} \mathrm{O}_{18}$, confirmed by the NMR spectral data (Table 8). Its IR absorption bands indicated the presence of hydroxyl $\left(3387 \mathrm{~cm}^{-1}\right), \alpha, \beta$-unsaturated ketone carbonyl $\left(1669 \mathrm{~cm}^{-1}\right)$, olefin $\left(1637 \mathrm{~cm}^{-1}\right)$, and an ether function $\left(1077 \mathrm{~cm}^{-1}\right)$. Its ${ }^{1} \mathrm{H}$ - and ${ }^{13} \mathrm{C}-\mathrm{NMR}$ spectra showed that the signals belonged to two $\beta$-D-glucopyranosyl $\left[84.95\left(1 \mathrm{H}, \mathrm{d}, J=7.5 \mathrm{~Hz}, \mathrm{H}-1^{\prime}\right), 5.10\left(1 \mathrm{H}, \mathrm{d}, J=8.0 \mathrm{~Hz}, \mathrm{H}-1^{\prime \prime}\right)\right]$, and one $\alpha$-L-arabinofuranosyl [ $\left.\delta 5.71\left(1 \mathrm{H}, \mathrm{d}, J=1.5 \mathrm{~Hz}, \mathrm{H}-1^{\prime \prime \prime}\right)\right]$. Its ${ }^{13} \mathrm{C}-\mathrm{NMR}$ spectrum displayed forty-seven carbons including thirty for aglycone and seventeen for sugar moieties. The ${ }^{1} \mathrm{H}$ and ${ }^{13} \mathrm{C}-\mathrm{NMR}$ spectra denoted that 8 was a dammarane-type triterpene saponin derivative. Compared to 1, the chemical shifts of proton and carbon for the side chain changed significantly, as follows-one disubstituted terminal olefin signals [85.78, 6.37 (1H each, both br. s, $\left.\left.\mathrm{H}_{2}-26\right)\right]$ appeared, while the signals of one methyl and one trans-olefinic bond disappeared in its ${ }^{1} \mathrm{H}-\mathrm{NMR}$ spectrum; meanwhile, an additional terminal olefin $\left[\delta_{C} 125.5(\mathrm{C}-26), 144.4(\mathrm{C}-25)\right]$ and additional carbonyl group $\left[\delta_{\mathrm{C}} 202.6\right.$ (C-24)] signals were displayed in its ${ }^{13} \mathrm{C}$-NMR spectrum, but one methyl, one trans-olefinic bond, as well as oxygenated quaternary carbon signals disappeared. The ${ }^{1} \mathrm{H}-{ }^{1} \mathrm{H}$ COSY experiment on 8 indicated the presence of partial structures written in bold lines, as shown in Figure 2. Finally, in the $\mathrm{HMBC}$ experiment, the following long-range correlations from proton to carbon pairs were found: $\delta_{\mathrm{H}} 5.78,6.37\left(\mathrm{H}_{2}-26\right)$ to $\delta_{\mathrm{C}} 17.8(\mathrm{C}-27), 144.4(\mathrm{C}-25), 202.6(\mathrm{C}-24) ; \delta_{\mathrm{H}} 1.85\left(\mathrm{H}_{3}-27\right)$ to $\delta_{\mathrm{C}} 125.5(\mathrm{C}-26)$, 144.4 (C-25), 202.6 (C-24), then the conjunction position of carbonyl and terminal olefin were clarified. Since the chemical shifts of its aglycone were identical to those of ginsenoside III [14], its aglycone of was identified as $3 \beta, 12 \beta, 20(S)$-trihydroxy-dammar-25-en-24-one. Consequently, the structure of notoginsenoside NL- $C_{1}(8)$ was elucidated. 
Table 8. The ${ }^{1} \mathrm{H}$ and ${ }^{13} \mathrm{C}-\mathrm{NMR}$ data for 8 in $\mathrm{C}_{5} \mathrm{D}_{5} \mathrm{~N}$

\begin{tabular}{|c|c|c|c|c|c|}
\hline No. & $\delta_{C}$ & $\delta_{\mathrm{H}}(J$ in $\mathrm{Hz})$ & No. & $\delta_{\mathrm{C}}$ & $\delta_{\mathrm{H}}(J$ in $\mathrm{Hz})$ \\
\hline 1 & 39.2 & $0.77,1.56$ (both m) & 25 & 144.4 & - \\
\hline 2 & 26.8 & $\begin{array}{c}\text { 1.82(m, overlapped }) \\
2.23(\mathrm{~m})\end{array}$ & 26 & 125.5 & $5.78,6.37$ (both br. s) \\
\hline 3 & 89.0 & $3.37(\mathrm{dd}, 2.5,10.5 \mathrm{~Hz})$ & 27 & 17.8 & $1.85(\mathrm{~s})$ \\
\hline 4 & 39.7 & - & 28 & 28.2 & $1.31(\mathrm{~s})$ \\
\hline 5 & 56.4 & 0.72 (br. d, ca. 12) & 29 & 16.8 & $1.00(\mathrm{~s})$ \\
\hline 6 & 18.5 & $1.38,1.50$ (both $\mathrm{m}$, overlapped) & 30 & 17.4 & $0.98(\mathrm{~s})$ \\
\hline 7 & 35.1 & $1.19,1.47$ (both m) & $1^{\prime}$ & 107.0 & $4.95(\mathrm{~d}, 7.5 \mathrm{~Hz})$ \\
\hline 8 & 40.0 & - & $2^{\prime}$ & 75.8 & $4.06(\mathrm{dd}, 7.5,8.0)$ \\
\hline 9 & 50.2 & 1.37 (m, overlapped) & $3^{\prime}$ & 78.8 & $4.27(\mathrm{dd}, 8.0,9.0)$ \\
\hline 10 & 37.0 & -4 & $4^{\prime}$ & 71.9 & $4.23(\mathrm{dd}, 8.5,9.0)$ \\
\hline 11 & 30.8 & $1.51,1.97$ (both m, overlapped) & $5^{\prime}$ & 78.4 & $4.03(\mathrm{~m})$ \\
\hline 12 & 70.2 & $4.21(\mathrm{~m})$ & $6^{\prime}$ & 63.1 & $\begin{array}{l}4.40(\mathrm{dd}, 5.5,11.5) \\
4.61(\mathrm{dd}, 1.5,11.5)\end{array}$ \\
\hline 13 & 49.5 & $2.04(\mathrm{~m})$ & $1^{\prime \prime}$ & 98.0 & $5.10(\mathrm{~d}, 8.0)$ \\
\hline 14 & 51.5 & - & $2^{\prime \prime}$ & 74.9 & $3.95(\mathrm{dd}, 8.0,8.5)$ \\
\hline 15 & 30.7 & $0.99,1.53$ (both m, overlapped) & $3^{\prime \prime}$ & 79.4 & $4.19(\mathrm{dd}, 8.5,9.0)$ \\
\hline 16 & 26.7 & $1.36,1.82$ (both $\mathrm{m}$, overlapped) & $4^{\prime \prime}$ & 72.0 & $3.97(\mathrm{dd}, 9.0,9.0)$ \\
\hline 17 & 51.9 & $2.60(\mathrm{q}$ like, $c a .10)$ & $5^{\prime \prime}$ & 76.7 & $4.09(\mathrm{~m})$ \\
\hline 18 & 16.0 & $0.92(\mathrm{~s})$ & $6^{\prime \prime}$ & 68.8 & $\begin{array}{l}4.13(\mathrm{dd}, 5.0,11.0) \\
4.70 \text { (br. d, ca. } 11)\end{array}$ \\
\hline 19 & 16.3 & $0.80(\mathrm{~s})$ & $1^{\prime \prime \prime}$ & 110.3 & $5.71(\mathrm{~d}, 1.5)$ \\
\hline 20 & 83.2 & - & $2^{\prime \prime \prime}$ & 83.5 & $4.88(\mathrm{dd}, 1.5,4.5)$ \\
\hline 21 & 21.9 & $1.58(\mathrm{~s})$ & $3^{\prime \prime \prime}$ & 78.8 & $4.82(\mathrm{dd}, 4.5,4.5)$ \\
\hline 22 & 29.8 & $2.09,2.75$ (both m) & $4^{\prime \prime \prime}$ & 86.0 & $\begin{array}{c}4.76(\mathrm{~m}) \\
4.25(\mathrm{~m}, \text { overlapped })\end{array}$ \\
\hline 23 & 32.7 & $\begin{array}{l}3.17 \text { (ddd, } 6.5,10.5,16.5) \\
3.40(\mathrm{ddd}, 3.5,10.5,16.5)\end{array}$ & $5^{\prime \prime \prime}$ & 62.7 & $4.35(\mathrm{dd}, 3.0,12.0)$ \\
\hline 24 & 202.6 & - & & & \\
\hline
\end{tabular}

Notoginsenoside NL- $C_{2}(9)$ was obtained as a white powder with positive optical rotation $\left([\alpha]_{\mathrm{D}}^{25}\right.$ $+1.8, \mathrm{MeOH})$. The same molecular formula, $\mathrm{C}_{47} \mathrm{H}_{78} \mathrm{O}_{18}\left(\mathrm{~m} / \mathrm{z} 929.51221[\mathrm{M}-\mathrm{H}]^{-}\right.$; calcd. for $\mathrm{C}_{47} \mathrm{H}_{77} \mathrm{O}_{18}$, 929.51044) as that of $\mathbf{8}$ was revealed by the 0ESI-Q-Orbitrap MS analysis. After acid hydrolysis, D-glucose and D-xylose were given [12]. Its ${ }^{1} \mathrm{H}$ and ${ }^{13} \mathrm{C}-\mathrm{NMR}$ (Table 9) spectra were similar to those of 8. The main differences between them were one $\beta$-D-xylopyranosyl [ $\left.\delta 4.91\left(1 \mathrm{H}, \mathrm{d}, J=7.5 \mathrm{~Hz}, \mathrm{H}-1^{\prime \prime \prime}\right)\right]$ appeared, and one $\alpha$-L-arabinofuranosyl disappeared in 9. Finally, the $\beta$-D-xylopyranosyl was clarified to have connected with 6-position of $\beta$-D-glucopyranosyl substituted at C-20 by cross peak from $\delta_{\mathrm{H}}$ $4.91\left(\mathrm{H}-1^{\prime \prime \prime}\right)$ to $\delta_{\mathrm{C}} 70.1\left(\mathrm{C}-6^{\prime \prime}\right)$, showed in HMBC (Figure 2).

The molecular formula of notoginsenoside $\mathrm{NL}^{-} \mathrm{C}_{3}(\mathbf{1 0})$ was measured to be $\mathrm{C}_{53} \mathrm{H}_{88} \mathrm{O}_{23}(\mathrm{~m} / \mathrm{z}$ $1091.56445\left[\mathrm{M}-\mathrm{H}^{-}\right.$; calcd. for $\left.\mathrm{C}_{53} \mathrm{H}_{87} \mathrm{O}_{23}, 1091.56327\right)$ on an ESI-Q-Orbitrap MS spectrometer. Compared with 9, one more group data of $\beta$-D-glucopyranosyl $\left[\delta 5.39\left(1 \mathrm{H}, \mathrm{d}, J=7.5 \mathrm{~Hz}, \mathrm{H}-1^{\prime \prime}\right)\right]$ was presented in 10 (Table 10). Moreover, the carbon signals of $C-1^{\prime}$ and $C-2^{\prime}$ significantly shifted to higher and lower field $\left[\delta_{C} 75.8\left(C-2^{\prime}\right), 107.0\left(C-1^{\prime}\right)\right.$ for 9; $\delta_{C} 83.5\left(C-2^{\prime}\right), 105.1\left(C-1^{\prime}\right)$ for 10], respectively, which suggested that $\mathrm{C}-2^{\prime}$ was displaced by the glycosyl group. The long-range correlation from $\delta_{\mathrm{H}} 5.39\left(\mathrm{H}-1^{\prime \prime}\right)$ to $\delta_{\mathrm{C}} 83.5\left(\mathrm{C}-2^{\prime}\right)$ displayed in its HMBC experiment confirmed the above-mentioned $\beta$-D-glucopyranosyl linked with C-2'. 
Table 9. The ${ }^{1} \mathrm{H}$ and ${ }^{13} \mathrm{C}-\mathrm{NMR}$ data for 9 in $\mathrm{C}_{5} \mathrm{D}_{5} \mathrm{~N}$

\begin{tabular}{|c|c|c|c|c|c|}
\hline No. & $\delta_{\mathrm{C}}$ & $\delta_{\mathrm{H}}(J$ in $\mathrm{Hz})$ & No. & $\delta_{\mathrm{C}}$ & $\delta_{\mathrm{H}}(J$ in $\mathrm{Hz})$ \\
\hline 1 & 39.2 & $\begin{array}{l}0.80 \text { (m, overlapped }) \\
1.58 \text { (m, overlapped })\end{array}$ & 25 & 144.4 & - \\
\hline 2 & 26.8 & $\begin{array}{c}1.84(\mathrm{~m}, \text { overlapped }) \\
2.24(\mathrm{~m})\end{array}$ & 26 & 125.5 & $5.72,6.37$ (both br. s) \\
\hline 3 & 88.9 & $3.38(\mathrm{dd}, 4.5,11.5)$ & 27 & 17.9 & $1.84(\mathrm{~s})$ \\
\hline 4 & 39.7 & - & 28 & 28.2 & $1.31(\mathrm{~s})$ \\
\hline 5 & 56.4 & 0.73 (br. d, ca. 12) & 29 & 16.8 & $1.00(\mathrm{~s})$ \\
\hline 6 & 18.5 & $\begin{array}{l}1.39(\mathrm{~m}, \text { overlapped }) \\
1.51(\mathrm{~m}, \text { overlapped })\end{array}$ & 30 & 17.4 & $0.98(\mathrm{~s})$ \\
\hline 7 & 35.1 & $1.20,1.48$ (both m) & $1^{\prime}$ & 107.0 & $4.95(\mathrm{~d}, 8.0 \mathrm{~Hz})$ \\
\hline 8 & 40.1 & - & $2^{\prime}$ & 75.8 & $4.05(\mathrm{dd}, 8.0,8.5)$ \\
\hline 9 & 50.3 & 1.37 (m, overlapped) & $3^{\prime}$ & 78.8 & $4.26(\mathrm{dd}, 8.5,8.5)$ \\
\hline 10 & 37.0 & - & $4^{\prime}$ & 71.9 & $4.22(\mathrm{dd}, 8.5,9.0)$ \\
\hline 11 & 30.8 & $\begin{array}{c}1.50(\mathrm{~m}, \text { overlapped }) \\
1.96(\mathrm{~m})\end{array}$ & $5^{\prime}$ & 78.4 & $4.02(\mathrm{~m}$, overlapped $)$ \\
\hline 12 & 70.1 & $4.21(\mathrm{~m})$ & $6^{\prime}$ & 63.1 & $\begin{array}{l}4.40(\mathrm{dd}, 5.5,11.0) \\
4.61(\mathrm{dd}, 2.0,11.5)\end{array}$ \\
\hline 13 & 49.5 & $2.03(\mathrm{dd}, 9.5,9.5)$ & $1^{\prime \prime}$ & 98.0 & $5.10(\mathrm{~d}, 7.5)$ \\
\hline 14 & 51.5 & - & $2^{\prime \prime}$ & 74.8 & $3.90(\mathrm{dd}, 7.5,8.0)$ \\
\hline 15 & 30.7 & $1.00,1.58$ (both m, overlapped) & $3^{\prime \prime}$ & 79.4 & $4.17(\mathrm{dd}, 8.0,8.5)$ \\
\hline 16 & 26.7 & $\begin{array}{l}\text { 1.37(m, overlapped) } \\
1.84 \text { (m, overlapped) }\end{array}$ & $4^{\prime \prime}$ & 71.3 & $4.12(\mathrm{dd}, 8.5,8.5)$ \\
\hline 17 & 52.1 & $2.60(\mathrm{q}$ like, $c a .10 \mathrm{~Hz})$ & $5^{\prime \prime}$ & 76.6 & $4.04(\mathrm{~m}$, overlapped $)$ \\
\hline 18 & 16.0 & $0.93(\mathrm{~s})$ & $6^{\prime \prime}$ & 70.1 & $\begin{array}{l}4.28(\mathrm{dd}, 5.5,11.5) \\
4.76(\mathrm{dd}, 2.0,11.5)\end{array}$ \\
\hline 19 & 16.3 & $0.82(\mathrm{~s})$ & $1^{\prime \prime \prime}$ & 106.1 & $4.91(\mathrm{~d}, 7.5)$ \\
\hline 20 & 83.3 & - & $2^{\prime \prime \prime}$ & 74.9 & $4.02(\mathrm{~m}$, overlapped $)$ \\
\hline 21 & 21.7 & $1.58(\mathrm{~s})$ & $3^{\prime \prime \prime}$ & 78.2 & $4.13(\mathrm{dd}, 8.5,8.5)$ \\
\hline 22 & 29.8 & $\begin{array}{c}2.11(\mathrm{~m}) \\
2.75(\mathrm{ddd}, 5.0,10.5,14.5)\end{array}$ & $4^{\prime \prime \prime}$ & 71.2 & $4.25(\mathrm{~m})$ \\
\hline 23 & 32.8 & $\begin{array}{l}3.18(\mathrm{ddd}, 6.5,10.0,17.5) \\
3.47(\mathrm{ddd}, 4.5,10.0,17.5)\end{array}$ & $5^{\prime \prime \prime}$ & 67.1 & $\begin{array}{c}3.69(\mathrm{dd}, 10.5,10.5) \\
4.37(\mathrm{dd}, 5.0,10.5)\end{array}$ \\
\hline 24 & 202.7 & - & & & \\
\hline
\end{tabular}

Table 10. The ${ }^{1} \mathrm{H}$ and ${ }^{13} \mathrm{C}-\mathrm{NMR}$ data for 10 in $\mathrm{C}_{5} \mathrm{D}_{5} \mathrm{~N}$

\begin{tabular}{|c|c|c|c|c|c|}
\hline No. & $\delta_{\mathrm{C}}$ & $\delta_{\mathrm{H}}(J$ in $\mathrm{Hz})$ & No. & $\delta_{\mathrm{C}}$ & $\delta_{\mathrm{H}}(J$ in $\mathrm{Hz})$ \\
\hline 1 & 39.3 & $\begin{array}{c}0.73(\mathrm{~m}) \\
1.54(\mathrm{~m}, \text { overlapped })\end{array}$ & 28 & 28.1 & $1.28(\mathrm{~s})$ \\
\hline 2 & 26.8 & $\begin{array}{c}1.83(\mathrm{~m} \text {, overlapped }) \\
2.20(\mathrm{~m})\end{array}$ & 29 & 16.6 & $1.11(\mathrm{~s})$ \\
\hline 3 & 89.0 & $3.28(\mathrm{dd}, 4.0,12.0)$ & 30 & 17.4 & $0.97(\mathrm{~s})$ \\
\hline 4 & 39.7 & - & $1^{\prime}$ & 105.1 & $4.92(\mathrm{~d}, 7.5)$ \\
\hline 5 & 56.4 & 0.66 (br. d, ca. 12) & $2^{\prime}$ & 83.5 & $4.26(\mathrm{dd}, 7.5,8.5)$ \\
\hline 6 & 18.4 & $\begin{array}{l}1.37(\mathrm{~m} \text {, overlapped }) \\
1.47(\mathrm{~m}, \text { overlapped })\end{array}$ & $3^{\prime}$ & 78.4 & $4.32(\mathrm{~m}$, overlapped $)$ \\
\hline 7 & 35.1 & $\begin{array}{c}1.18(\mathrm{~m}) \\
1.46(\mathrm{~m}, \text { overlapped })\end{array}$ & $4^{\prime}$ & 71.7 & 4.35 (m, overlapped) \\
\hline 8 & 40.0 & - & $5^{\prime}$ & 78.3 & $3.94\left(\mathrm{~m}, \mathrm{H}-5^{\prime}\right)$ \\
\hline 9 & 50.2 & $1.36(\mathrm{~m}$, overlapped $)$ & $6^{\prime}$ & 62.9 & $\begin{array}{c}4.35(\mathrm{~m}, \text { overlapped }) \\
4.57(\mathrm{dd}, 1.5,11.5)\end{array}$ \\
\hline 10 & 36.9 & - & $1^{\prime \prime}$ & 106.1 & $5.39(\mathrm{~d}, 7.5)$ \\
\hline 11 & 30.9 & $\begin{array}{c}1.54(\mathrm{~m}, \text { overlapped }) \\
1.96(\mathrm{~m})\end{array}$ & $2^{\prime \prime}$ & 77.2 & $4.14(\mathrm{dd}, 7.5,8.5)$ \\
\hline 12 & 70.1 & $4.19(\mathrm{~m})$ & $3^{\prime \prime}$ & 78.1 & $4.26(\mathrm{~m}$, overlapped $)$ \\
\hline 13 & 49.5 & $2.03(\mathrm{dd}, 10.5,10.5)$ & $4^{\prime \prime}$ & 71.7 & $4.14(\mathrm{dd}, 7.5,8.5)$ \\
\hline 14 & 51.5 & - & $5^{\prime \prime}$ & 78.2 & $3.93(\mathrm{~m})$ \\
\hline 15 & 30.7 & $\begin{array}{c}0.98(\mathrm{~m}) \\
1.54(\mathrm{~m} \text {, overlapped })\end{array}$ & $6^{\prime \prime}$ & 62.7 & $4.50(\mathrm{~m})$ \\
\hline 16 & 26.7 & $\begin{array}{l}1.37(\mathrm{~m}, \text { overlapped }) \\
1.83(\mathrm{~m} \text {, overlapped })\end{array}$ & $1^{\prime \prime \prime}$ & 98.0 & $5.10(\mathrm{~d}, 8.0)$ \\
\hline 17 & 52.1 & 2.58 (q like, $c a .11)$ & $2^{\prime \prime \prime}$ & 74.8 & $3.90(\mathrm{dd}, 8.0,8.5)$ \\
\hline 18 & 16.0 & $0.92(\mathrm{~s})$ & $3^{\prime \prime \prime}$ & 79.5 & $4.17(\mathrm{dd}, 8.5,8.5)$ \\
\hline 19 & 16.3 & $0.81(\mathrm{~s})$ & $4^{\prime \prime \prime}$ & 71.4 & $4.13(\mathrm{~m}$, overlapped $)$ \\
\hline 20 & 83.2 & - & $5^{\prime \prime \prime}$ & 76.6 & $4.05(\mathrm{~m})$ \\
\hline 21 & 21.7 & $1.58(\mathrm{~s})$ & $6^{\prime \prime \prime}$ & 70.2 & $\begin{array}{c}4.27(\mathrm{~m} \text {, overlapped }) \\
4.77(\mathrm{dd}, 1.5,11.5)\end{array}$ \\
\hline 22 & 29.8 & $\begin{array}{c}2.11(\mathrm{~m}) \\
2.75(\mathrm{ddd}, 4.0,9.0,16.5)\end{array}$ & $1^{\prime \prime \prime \prime}$ & 106.1 & $4.91(\mathrm{~d}, 7.5)$ \\
\hline 23 & 32.8 & $\begin{array}{l}3.18 \text { (ddd, } 5.5,9.0,16.5) \\
3.45 \text { (ddd, } 4.0,9.0,16.5)\end{array}$ & $2^{\prime \prime \prime \prime}$ & 74.9 & $4.02(\mathrm{dd}, 7.5,8.5)$ \\
\hline 24 & 202.6 & - & $3^{\prime \prime \prime \prime}$ & 78.0 & 4.14 (m, overlapped) \\
\hline 25 & 144.4 & - & $4^{\prime \prime \prime \prime}$ & 71.2 & $4.25(\mathrm{dd}, 8.0,8.5)$ \\
\hline 26 & 125.5 & $5.51,5.71$ (both br. s) & $5^{\prime \prime \prime \prime}$ & 67.2 & $\begin{array}{c}3.69(\mathrm{dd}, 10.5,10.5) \\
4.36(\mathrm{~m} \text {, overlapped })\end{array}$ \\
\hline 27 & 17.9 & $1.83(\mathrm{~s})$ & & & \\
\hline
\end{tabular}


Notoginsenoside NL-D (11) was a white powder with positive optical rotation $\left([\alpha]_{\mathrm{D}}^{25}+29.6, \mathrm{MeOH}\right)$. The molecular formula, $\mathrm{C}_{44} \mathrm{H}_{72} \mathrm{O}_{16}$ of $\mathbf{1 1}$ was determined by ESI-Q-Orbitrap MS $(\mathrm{m} / \mathrm{z} 855.47388$ [M $-\mathrm{H}]^{-}$; calcd. for $\mathrm{C}_{44} \mathrm{H}_{71} \mathrm{O}_{16}, 855.47366$ ). The IR absorptions at 3382, 1717, 1649, and $1075 \mathrm{~cm}^{-1}$ indicated the presence of $\mathrm{OH}, \mathrm{CO}$, olefin, and an ether group. The ${ }^{1} \mathrm{H}$ and ${ }^{13} \mathrm{C}-\mathrm{NMR}$ (Table 11) and 2D NMR spectra, including ${ }^{1} \mathrm{H}-{ }^{1} \mathrm{H}$ COSY, HSQC, and HMBC suggested that $\mathbf{1 1}$ had the same sugar moiety, $\beta$-D-arabinopyranosyl $(1 \rightarrow 6)$ - $\beta$-D-glucopyranosyl $\left[\delta 5.00\left(1 \mathrm{H}, \mathrm{d}, J=5.5 \mathrm{~Hz}, \mathrm{H}-1^{\prime \prime \prime}\right), 5.12(1 \mathrm{H}\right.$, $\left.\left.\mathrm{d}, J=7.5 \mathrm{~Hz}, \mathrm{H}-1^{\prime \prime}\right)\right]$ and malonyl $\left[\delta_{\mathrm{C}} 43.8\left(\mathrm{C}-2^{\prime}\right), 168.4\left(\mathrm{C}-1^{\prime}\right), 170.6\left(\mathrm{C}-3^{\prime}\right)\right]$ as 4 . Forty-four signals were observed in its ${ }^{13} \mathrm{C}-\mathrm{NMR}$ spectrum. Except for the signals belonging to the above-mentioned moieties, the other 30 indicated that 11 was a triterpene saponin. Its ${ }^{1} \mathrm{H}-\mathrm{NMR}$ spectrum showed eight methyl signals at $\delta 0.93,0.97,1.05,1.35,1.62,1.63,1.66$, and 1.76 ( $3 \mathrm{H}$ each, all s, $\mathrm{H}_{3}-19,30,18,29,26,21$, $27,28)$, three methines bearing an oxygen function at $84.19(1 \mathrm{H}, \mathrm{m}, \mathrm{H}-12), 4.32(1 \mathrm{H}, \mathrm{m}$, overlapped, $\mathrm{H}-6)$, and $4.88(1 \mathrm{H}, \mathrm{dd}, J=4.0,10.5 \mathrm{~Hz}, \mathrm{H}-3)$, one trisubstituted olefin signal at $\delta 5.32(1 \mathrm{H}, \mathrm{t}, J=6.5 \mathrm{~Hz}$, $\mathrm{H}-24)$. In its $\mathrm{HMBC}$ experiment, long-range correlations were observed from the following proton to carbon pairs: $\mathrm{H}-3$ to $\mathrm{C}-\mathrm{1}^{\prime} ; \mathrm{H}_{3}-18$ to C-7-9, C-14; $\mathrm{H}_{3}-19$ to $\mathrm{C}-1, \mathrm{C}-5, \mathrm{C}-9, \mathrm{C}-10 ; \mathrm{H}_{3}-21$ to C-17, C-20, C-22; $\mathrm{H}_{3}-26$ to $\mathrm{C}-24, \mathrm{C}-25, \mathrm{C}-27 ; \mathrm{H}_{3}-27$ to $\mathrm{C}-24-26 ; \mathrm{H}_{3}-28$ to $\mathrm{C}-3-5, \mathrm{C}-29 ; \mathrm{H}_{3}-29$ to $\mathrm{C}-3-5, \mathrm{C}-28$; and $\mathrm{H}_{3}-30$ to $\mathrm{C}-8, \mathrm{C}-13-15 ; \mathrm{H}-1^{\prime \prime}$ to $\mathrm{C}-20$. Then, its planar structure was elucidated, which was a protopanaxatriol type saponin. The chemical shifts for proton and carbon of the B-D ring and the side chain were matched closely with those of ginsenoside $U$ [15]. Since they were more different in 1-4 positions of two compounds, they might have been affected by the configuration of $\mathrm{C}-3$ or a malonyl substitution. Finally, the NOE correlations between $\delta_{\mathrm{H}} 4.19(\mathrm{H}-12)$ and $\delta_{\mathrm{H}} 1.49(\mathrm{H}-9) ; \delta_{\mathrm{H}} 1.18(\mathrm{H}-5)$ and $\delta_{\mathrm{H}} 1.49$ (H-9), 4.88 (H-3) displayed in its NOESY experiment (Figure 2) denoted that $\mathrm{H}-3$ showed $\alpha$-orientation. Thus, the structure of notoginsenoside NL-D (11) was determined.

Table 11. The ${ }^{1} \mathrm{H}$ and ${ }^{13} \mathrm{C}-\mathrm{NMR}$ data for 11 in $\mathrm{C}_{5} \mathrm{D}_{5} \mathrm{~N}$

\begin{tabular}{|c|c|c|c|c|c|}
\hline No. & $\delta_{C}$ & $\delta_{H}(J$ in $\mathrm{Hz})$ & No. & $\delta_{\mathrm{C}}$ & $\delta_{H}(J$ in $\mathrm{Hz})$ \\
\hline 1 & 38.5 & $0.89,1.61$ (both m) & 23 & 23.2 & $2.34,2.58$ (both $\mathrm{m}$ ) \\
\hline 2 & 23.8 & $1.80(\mathrm{~m})$ & 24 & 125.9 & $5.32(t, 6.5)$ \\
\hline 3 & 82.1 & $4.88(\mathrm{dd}, 4.0,10.5)$ & 25 & 131.1 & \\
\hline 4 & 39.1 & & 26 & 25.8 & $1.62(\mathrm{~s})$ \\
\hline 5 & 61.4 & $1.18(\mathrm{~d}, 10.0)$ & 27 & 17.9 & $1.66(\mathrm{~s})$ \\
\hline 6 & 67.3 & $4.32(\mathrm{~m}$, overlapped $)$ & 28 & 31.3 & $1.76(\mathrm{~s})$ \\
\hline 7 & 47.2 & $\begin{array}{c}1.83(\mathrm{~m}, \text { overlapped }) \\
1.93(\mathrm{dd}, 11.0,11.0)\end{array}$ & 29 & 17.0 & $1.35(\mathrm{~s})$ \\
\hline 8 & 41.1 & & 30 & 17.4 & $0.97(\mathrm{~s})$ \\
\hline 9 & 49.6 & 1.49 (m, overlapped) & $1^{\prime}$ & 168.4 & \\
\hline 10 & 39.0 & & $2^{\prime}$ & 43.8 & $3.83(\mathrm{~s})$ \\
\hline 11 & 30.7 & $\begin{array}{c}1.49(\mathrm{~m}, \text { overlapped }) \\
1.99(\mathrm{~m})\end{array}$ & $3^{\prime}$ & 170.6 & \\
\hline 12 & 70.1 & $4.19(\mathrm{~m})$ & $1^{\prime \prime}$ & 98.1 & $5.12(\mathrm{~d}, 7.5)$ \\
\hline 13 & 49.1 & 1.97 (m, overlapped) & $2^{\prime \prime}$ & 74.9 & $3.94(\mathrm{dd}, 7.5,7.5)$ \\
\hline 14 & 51.3 & & $3^{\prime \prime}$ & 79.2 & $4.18(\mathrm{dd}, 7.5,9.0)$ \\
\hline 15 & 30.7 & $\begin{array}{c}0.97(\mathrm{~m}, \text { overlapped }) \\
1.55(\mathrm{~m})\end{array}$ & $4^{\prime \prime}$ & 71.8 & $4.07(\mathrm{dd}, 9.0,10.0)$ \\
\hline 16 & 26.6 & $\begin{array}{c}1.31(\mathrm{~m}) \\
1.81(\mathrm{~m}, \text { overlapped })\end{array}$ & $5^{\prime \prime}$ & 76.7 & $4.05(\mathrm{~m})$ \\
\hline 17 & 51.6 & 2.55 (q like, $c a .10)$ & $6^{\prime \prime}$ & 69.3 & $\begin{array}{l}4.26(\mathrm{dd}, 5.0,10.5) \\
4.70 \text { (br. d, ca. } 11)\end{array}$ \\
\hline 18 & 17.5 & $1.05(\mathrm{~s})$ & $1^{\prime \prime \prime}$ & 104.7 & $5.00(\mathrm{~d}, 5.5)$ \\
\hline 19 & 17.3 & $0.93(\mathrm{~s})$ & $2^{\prime \prime \prime}$ & 72.2 & $4.46(\mathrm{dd}, 5.5,7.0)$ \\
\hline 20 & 83.4 & & $3^{\prime \prime \prime}$ & 74.1 & $4.23(\mathrm{dd}, 3.0,7.0)$ \\
\hline 21 & 22.2 & $1.63(\mathrm{~s})$ & $4^{\prime \prime \prime}$ & 68.6 & $4.37(\mathrm{~m})$ \\
\hline 22 & 36.1 & $\begin{array}{c}1.81(\mathrm{~m}, \text { overlapped }) \\
2.39(\mathrm{~m})\end{array}$ & $5^{\prime \prime \prime}$ & 65.7 & $\begin{array}{l}3.79 \text { (br. d, ca. 12) } \\
4.32(\mathrm{dd}, 3.0,12.0)\end{array}$ \\
\hline
\end{tabular}

Moreover, in order to clarify the anti-inflammatory effects of 1-11, their inhibitory activities on LPS-stimulated NO release were measured using the method reported previously in [16].

Before the experiment, dimethyl thiazolyl diphenyl tetrazolium (MTT) assay was used to test the cytotoxicities of 1-11. It was found that compounds 1, 2, 5, 6, 8-10 displayed no significant cytotoxicity at $50 \mu \mathrm{M}$ concentration (Figure S74). Then, under this concentration, in vitro potential 
anti-inflammatory effects of all isolates were investigated. As a result, all of them exhibited significant inhibitory effects of the NO release (Table 12).

Table 12. Inhibitory effects of $\mathbf{1}, \mathbf{2}, \mathbf{5}, \mathbf{6}$, and $\mathbf{8}-\mathbf{1 0}$ on NO production in RAW 264.7 cells.

\begin{tabular}{cccc}
\hline NO. & NRC (\%) & NO. & NRC (\%) \\
\hline Normal & $3.2 \pm 0.6$ & 5 & $36.8 \pm 4.1^{* * *}$ \\
Control & $100 \pm 4.2$ & 6 & $51.3 \pm 5.8^{* * *}$ \\
DEX & $75.3 \pm 2.6^{* * *}$ & 8 & $44.6 \pm 4.8^{* * *}$ \\
1 & $62.4 \pm 8.0^{* * *}$ & 9 & $30.1 \pm 3.0^{* * *}$ \\
2 & $33.1 \pm 1.2^{* * *}$ & 10 & $39.4 \pm 1.9^{* * *}$
\end{tabular}

Positive control: Dexamethasone (Dex). Nitrite relative concentration (NRC): percentage of control group (set as $100 \%$ ). Values represent the mean \pm SD of three determinations. ${ }^{* * *} p<0.001$ (Differences between compound-treated group and control group), $N=4$. Final concentration was $50 \mu \mathrm{M}$ for compounds $\mathbf{1}, \mathbf{2}, \mathbf{5}, \mathbf{6}, \mathbf{8 - 1 0}$, and $1.0 \mu \mathrm{g} / \mathrm{mL}$ for positive control (Dex), respectively.

Anti-inflammatory activities of 1, 2, 5, 6, and 8-10 suggested that the number and type of substituted glycosyls mattered a lot for the anti-inflammatory activity of triterpenoids-the activities of $\beta$-D-xylopyranosyl-substituted triterpenoids were higher than the ones tha were $\alpha$-L-arabinofuranosyl-substituted (1 vs. 2; 8 vs. 9); Meanwhile, with an increasing number of $\beta$-D-glucopyranosyl, a stronger activity appeared ( 9 vs. 10). Additionally, the different side chains of C-20 displayed a strong effect on their activities (1 vs. 8; 2 vs. 9).

Moreover, a dose-dependent experiment was performed for the above-mentioned compounds at concentrations of 10, 25, and $50 \mu \mathrm{M}$, respectively. All of them were found to inhibit NO release from RAW264.7 cells in a dose-dependent manner (Figure 3).
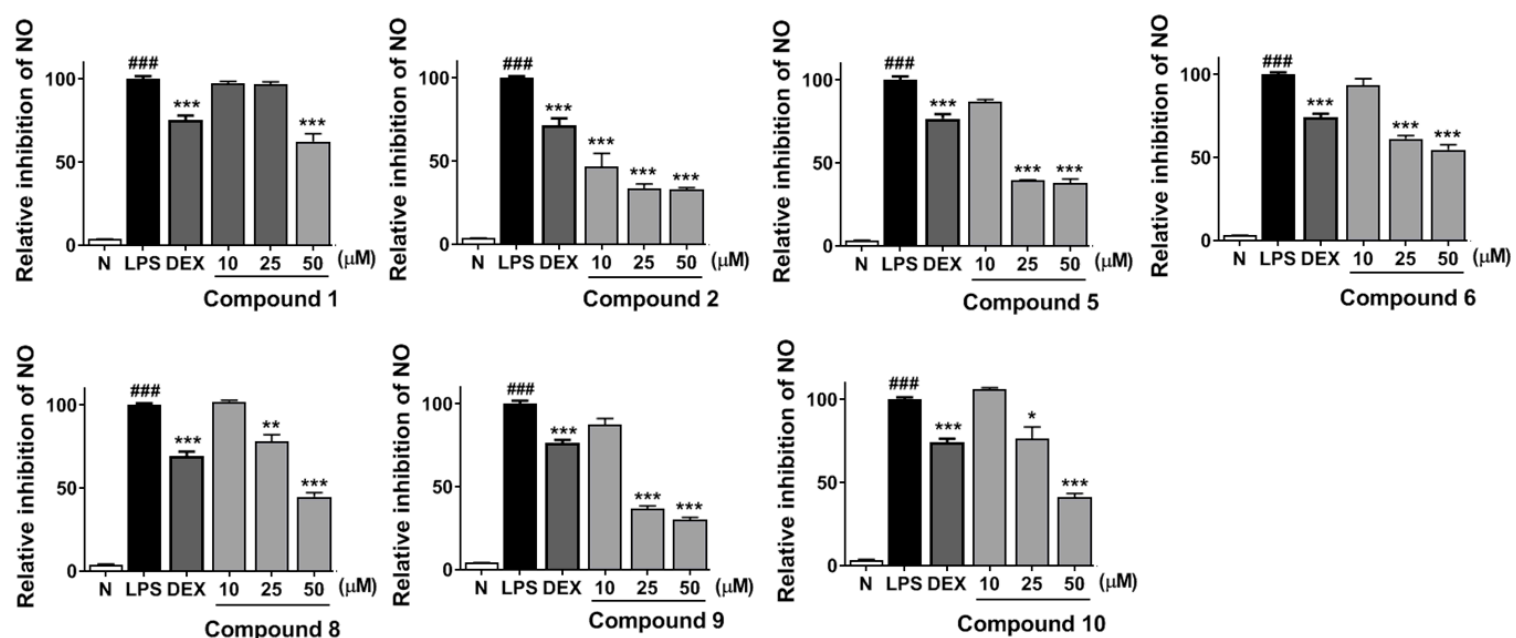

Figure 3. Inhibitory effects of compounds $\mathbf{1}, \mathbf{2}, \mathbf{5}, \mathbf{6}, \mathbf{8 - 1 0}$ at concentrations of 10, 25, and $50 \mu \mathrm{M}$ on $\mathrm{NO}$ production in RAW 264.7 cells, respectively. Nitrite relative concentration (NRC)-percentage of control group (set as $100 \%$ ). Values represent the mean $\pm \mathrm{SD}$ of four determinations. ${ }^{*} p<0.05,{ }^{* *} p<0.01$, *** $p<0.001$ vs. LPS group; \#\#\# $p<0.001$ vs normal group $(N)$ Differences between compound-treated group and control group; $N=4$.

\section{Materials and Methods}

\subsection{Experimental Procedures for Phytochmistry Study}

\subsubsection{General Experimental Procedures}

The following instruments were used to obtain physical data-NMR spectra were measured on Bruker ascend $600 \mathrm{MHz}$ or a Bruker ascend $500 \mathrm{MHz}$ NMR spectrometer (Bruker BioSpin AG 
Industriestrasse $26 \mathrm{CH}-8117$, Fällanden, Switzerland) with tetramethylsilane as an internal standard. The negative-ion mode ESI-Q-Orbitrap MS was obtained on a Thermo UltiMate 3000 UHPLC instrument (Thermo, Waltham, MA, USA). Optical rotations, UV, and IR spectra were run on a Rudolph Autopol ${ }^{\circledR}$ IV automatic polarimeter $(1=50 \mathrm{~mm})$ (Rudolph Research Analytical, Hackettstown NJ, USA), Varian Cary 50 UV-Vis (Varian, Inc., Hubbardsdon, MA, USA) and Varian 640-IR FT-IR spectrophotometer (Varian Australia Pty Ltd., Mulgrave, Australia), respectively.

CC were performed on macroporous resin D101 (Haiguang Chemical Co., Ltd., Tianjin, China),

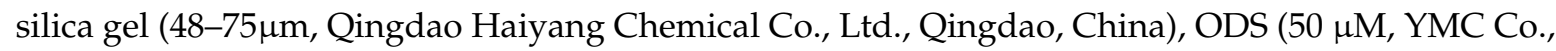
Ltd., Tokyo, Japan), MCI gel (Mitsubishi Chemical Corporation, Osaka, Japan), and Sephadex LH-20 (Ge Healthcare Bio-Sciences, Uppsala, Sweden). High performance liquid chromatography (HPLC) column-Cosmosil 5C 18 -MS-II (4.6 mm i.d. $\times 250 \mathrm{~mm}, 5 \mu \mathrm{M})$ and Cosmosil 5C 18 -MS-II $(20 \mathrm{mmi} . \mathrm{d} . \times$ $250 \mathrm{~mm}, 5 \mu \mathrm{M}$, NakalaiTesque, Inc., Tokyo, Japan) were used to analyze and separate the constituents.

\subsubsection{Plant Material}

The leaves of Panax notoginseng (Burk.) F. H. Chen were collected from Shilin country, Kunming city, Yunnan province, China, and identified by Dr. Wang Tao (Institute of Traditional Chinese Medicine, Tianjin University of Traditional Chinese Medicine). The voucher specimen was deposited at the Academy of Traditional Chinese Medicine of Tianjin University of TCM.

\subsubsection{Extraction and Isolation}

The dried leaves of $P$. notoginseng $(8 \mathrm{~kg}$ ) was extracted three times with $50 \% \mathrm{EtOH}$ under reflux for $3 \mathrm{~h}, 2 \mathrm{~h}$, and 2 h, successively. Evaporation of the solvent under reduced pressure provided the $50 \%$ EtOH extract $(2.67 \mathrm{~kg})$. Then, an aliquot $(2.1 \mathrm{~kg})$ of the $50 \%$ EtOH extract was subjected to D101 resin $\mathrm{CC}\left(\mathrm{H}_{2} \mathrm{O} \rightarrow 95 \% \mathrm{EtOH}\right)$ to give $\mathrm{H}_{2} \mathrm{O}-(760.0 \mathrm{~g})$ and $95 \%$ EtOH eluted fraction (695.0 g), respectively.

The $95 \%$ EtOH eluate $(150.0 \mathrm{~g})$ was separated by silica gel CC $\left[\mathrm{CH}_{2} \mathrm{Cl}_{2} \rightarrow \mathrm{CH}_{2} \mathrm{Cl}_{2}-\mathrm{MeOH}(100: 3\right.$ $\rightarrow 100: 7 \rightarrow 10: 1 \rightarrow 8: 1 \rightarrow 3: 1 \rightarrow 2: 1 \rightarrow 1: 1, v / v) \rightarrow \mathrm{MeOH}]$, and 12 fractions (Fr. 1-Fr. 12) were yielded. Fraction 6 (6.2 g) was further subjected to silica gel CC $\left[\mathrm{CH}_{2} \mathrm{Cl}_{2}-\mathrm{MeOH}-\mathrm{H}_{2} \mathrm{O}(40: 3: 1 \rightarrow\right.$ 30:3:1 $\rightarrow$ 25:3:1 $\rightarrow$ 15:3:1 $\rightarrow$ 13:3:1 $\rightarrow$ 12:3:1 $\rightarrow$ 10:3:1 $\rightarrow$ 8:3:1 $\rightarrow$ 6:3:1, v/v/v, lower layer $\rightarrow$ MeOH] to give 13 fractions (Fr. 6-1-Fr. 6-13). Fraction 6-5 (650.0 mg) was isolated by pHPLC [CH $\mathrm{CH}_{3} \mathrm{CN}$ \% HAc $\left.(37: 63, v / v)\right]$ to gain notoginsenoside NL-A 4 (4, 8.1 mg). Fraction 6-10 $(800.0 \mathrm{mg})$ was purified by pPHPLC $\left[\mathrm{CH}{ }_{3} \mathrm{CN}-1 \%\right.$ HAc $(37: 63, v / v)]$, and notoginsenosides NL-A 1 (1, $44.9 \mathrm{mg}), \mathrm{NL}_{-} \mathrm{C}_{1}(\mathbf{8}, 12.4 \mathrm{mg})$, as well as NL-D (11, $32.9 \mathrm{mg})$ were given. Fraction $7(30.0 \mathrm{~g})$ was separated by $\mathrm{MCI}$ gel CC $\left[\mathrm{MeOH}-\mathrm{H}_{2} \mathrm{O}(65 \% \rightarrow 70 \%\right.$ $\rightarrow 75 \% \rightarrow 80 \% \rightarrow 100 \%, v / v)]$ to provide 12 fractions (Fr. 7-1-Fr. 7-12). Fraction 7-5 (480.0 mg) was further isolated by pHPLC $\left[\mathrm{CH}_{3} \mathrm{CN}-1 \% \operatorname{HAc}(30: 70, v / v)\right]$, and notoginsenoside NL-B 1 (5, $\left.23.8 \mathrm{mg}\right)$ was yielded. Fraction 7-6 (800.0 mg) was subjected to pHPLC [MeOH-1\% HAc (70:30, v/v)] to give seven fractions (Fr. 7-6-1-Fr. 7-6-7). Fraction 7-6-3 (141.5 mg) was purified by pHPLC $\left[\mathrm{CH}_{3} \mathrm{CN}-1 \%\right.$ HAc $(35: 65, v / v)]$ to gain notoginsenoside NL-C $2(9,11.9 \mathrm{mg})$. Fraction 7-6-4 (179.5 mg) was isolated by pHPLC $\left[\mathrm{CH}_{3} \mathrm{CN}-1 \%\right.$ HAc $\left.(32: 68, v / v)\right]$ to produce notoginsenoside NL-A 2 (2, $\left.8.0 \mathrm{mg}\right)$. Fraction 8 (25.0 g) was subjected to $\mathrm{MCI}$ gel CC $\left[\mathrm{MeOH}-\mathrm{H}_{2} \mathrm{O}(40 \% \rightarrow 60 \% \rightarrow 70 \% \rightarrow 80 \% \rightarrow 100 \%, v / v)\right]$, as a result, 11 fractions (Fr. 8-1-Fr. 8-11) were gained. Fraction 8-6 (1.6 g) was separated by pHPLC [MeOH-1\% Hac $(60: 40, v / v)]$ to yield 13 fractions (Fr. 8-6-1-Fr. 8-6-13). Fraction 8-6-6 (135.3 mg) was further purified by $\mathrm{pHPLC}\left[\mathrm{CH}_{3} \mathrm{CN}-1 \% \mathrm{HAc}(26: 74, v / v)\right]$ to obtain notoginsenosides NL-A $3(3,31.2 \mathrm{mg})$ and NL-B 2 $(6,10.2 \mathrm{mg})$. Fraction 8-6-9 (223.8 mg) was isolated by pHPLC $\left[\mathrm{CH}_{3} \mathrm{CN}-1 \% \mathrm{HAc}(27: 73, v / v)\right]$ to gain notoginsenoside $\mathrm{NL}^{-\mathrm{C}_{3}}(\mathbf{1 0}, 37.4 \mathrm{mg})$. Fraction $9(15.0 \mathrm{~g})$ was subjected to MCI CC $\left[\mathrm{MeOH}-\mathrm{H}_{2} \mathrm{O}(60 \%\right.$ $\rightarrow 70 \% \rightarrow 80 \% \rightarrow 100 \%, v / v)]$, and 10 fractions (Fr. 9-1-Fr. 9-10) were given. Fraction 9-4 (500.0 mg) was separated by pHPLC $\left[\mathrm{CH}_{3} \mathrm{CN}-1 \% \operatorname{HAc}(26: 74, v / v)\right]$ to yield 11 fractions (Fr. 9-4-1-Fr. 9-4-10). Fraction 9-4-10 (10.1 mg) was further purified by pHPLC [MeOH-1\% HAc $(60: 40, v / v)]$, and notoginsenoside $\mathrm{NL}_{3}(7,5.6 \mathrm{mg})$ was gained.

Notoginsenoside $N L-A_{1}(\mathbf{1})$ : White powder; $[\alpha]_{\mathrm{D}}^{25}-1.8$ (conc. $\left.0.87, \mathrm{MeOH}\right) ; \mathrm{IR}(\mathrm{KBr}) v_{\max } 3368,2942$, 2877, 1635, 1455, 1384, 1075, 1037, $895 \mathrm{~cm}^{-1} ;{ }^{1} \mathrm{H}-\mathrm{NMR}\left(\mathrm{C}_{5} \mathrm{D}_{5} \mathrm{~N}, 500 \mathrm{MHz}\right)$ and ${ }^{13} \mathrm{C}-\mathrm{NMR}\left(\mathrm{C}_{5} \mathrm{D}_{5} \mathrm{~N}\right.$, 
$125 \mathrm{MHz}$ ) spectroscopic data-see Table 1; ESI-Q-Orbitrap MS m/z 947.52405 [M - H] ${ }^{-}$(calcd. for $\mathrm{C}_{47} \mathrm{H}_{79} \mathrm{O}_{19}$, 947.52101).

Notoginsenoside $N L-A_{2}$ (2): White powder; $[\alpha]_{\mathrm{D}}^{25}+6.0$ (conc. 0.26, MeOH); IR (KBr) $v_{\max } 3418,2968$, $2865,1647,1455,1395,1052,1033,1014 \mathrm{~cm}^{-1} ;{ }^{1} \mathrm{H}-\mathrm{NMR}\left(\mathrm{C}_{5} \mathrm{D}_{5} \mathrm{~N}, 500 \mathrm{MHz}\right)$ and ${ }^{13} \mathrm{C}-\mathrm{NMR}\left(\mathrm{C}_{5} \mathrm{D}_{5} \mathrm{~N}\right.$, $125 \mathrm{MHz}$ ) spectroscopic data-see Table 2; ESI-Q-Orbitrap MS m/z $947.51996[\mathrm{M}-\mathrm{H}]^{-}$(calcd. for $\mathrm{C}_{47} \mathrm{H}_{79} \mathrm{O}_{19}$, 947.52101).

Notoginsenoside $N L-A_{3}$ (3): White powder; $[\alpha]_{\mathrm{D}}^{25}-15.0$ (conc. 0.80, MeOH); IR (KBr) $v_{\max } 3368$, 2939, 2877, 1635, 1453, 1386, 1074, $1042 \mathrm{~cm}^{-1},{ }^{1} \mathrm{H}-\mathrm{NMR}\left(\mathrm{C}_{5} \mathrm{D}_{5} \mathrm{~N}, 500 \mathrm{MHz}\right)$ and ${ }^{13} \mathrm{C}-\mathrm{NMR}\left(\mathrm{C}_{5} \mathrm{D}_{5} \mathrm{~N}\right.$, $125 \mathrm{MHz}$ ) spectroscopic data-see Table 3; ESI-Q-Orbitrap MS m/z 1241.61584 [M - H] ${ }^{-}$(calcd. for $\left.\mathrm{C}_{58} \mathrm{H}_{97} \mathrm{O}_{28}, 1241.61609\right)$.

Notoginsenoside $N L-A_{4}(4)$ : White powder; $[\alpha]_{\mathrm{D}}^{25}+18.7$ (conc. $\left.0.31, \mathrm{MeOH}\right) ; \mathrm{IR}(\mathrm{KBr}) v_{\max } 3379$, 2946, 2877, 1718, 1646, 1456, 1384, 1080, 1041, $1010 \mathrm{~cm}^{-1} ;{ }^{1} \mathrm{H}-\mathrm{NMR}\left(\mathrm{C}_{5} \mathrm{D}_{5} \mathrm{~N}, 500 \mathrm{MHz}\right)$ and ${ }^{13} \mathrm{C}-\mathrm{NMR}$ $\left(\mathrm{C}_{5} \mathrm{D}_{5} \mathrm{~N}, 125 \mathrm{MHz}\right.$ ) spectroscopic data-see Table 4; ESI-Q-Orbitrap MS m/z 871.46875 [M - H] ${ }^{-}$(calcd. for $\mathrm{C}_{44} \mathrm{H}_{71} \mathrm{O}_{17}, 871.46858$ ).

Notoginsenoside $N L-B_{1}$ (5): White powder; $[\alpha]_{\mathrm{D}}^{25}+2.2$ (conc. 1.19, MeOH); IR (KBr) $v_{\max } 3379,2944$, $2877,1649,1458,1387,1076,1035,1018 \mathrm{~cm}^{-1} ;{ }^{1} \mathrm{H}-\mathrm{NMR}\left(\mathrm{C}_{5} \mathrm{D}_{5} \mathrm{~N}, 500 \mathrm{MHz}\right)$ and ${ }^{13} \mathrm{C}-\mathrm{NMR}\left(\mathrm{C}_{5} \mathrm{D}_{5} \mathrm{~N}\right.$, $125 \mathrm{MHz}$ ) spectroscopic data-see Table 5; ESI-Q-Orbitrap MS m/z $931.52838[\mathrm{M}-\mathrm{H}]^{-}$(calcd. for $\mathrm{C}_{47} \mathrm{H}_{79} \mathrm{O}_{18}$, 931.52609).

Notoginsenoside $N L-B_{2}$ (6): White powder; $[\alpha]_{\mathrm{D}}^{25}-2.1$ (conc. $\left.0.28, \mathrm{MeOH}\right) ; \mathrm{IR}(\mathrm{KBr}) v_{\max } 3364$, 2938, 2877, 1647, 1456, 1384, 1073, 1042, $893 \mathrm{~cm}^{-1} ;{ }^{1} \mathrm{H}-\mathrm{NMR}\left(\mathrm{C}_{5} \mathrm{D}_{5} \mathrm{~N}, 500 \mathrm{MHz}\right)$ and ${ }^{13} \mathrm{C}-\mathrm{NMR}\left(\mathrm{C}_{5} \mathrm{D}_{5} \mathrm{~N}\right.$, $125 \mathrm{MHz}$ ) spectroscopic data-see Table 6; ESI-Q-Orbitrap MS m/z 1225.62134 [M - H] $]^{-}$(calcd. for $\left.\mathrm{C}_{58} \mathrm{H}_{97} \mathrm{O}_{27}, 1225.62117\right)$.

Notoginsenoside NL- $B_{3}$ (7): White powder; $[\alpha]_{\mathrm{D}}^{25}-1.7$ (conc. 0.23, MeOH); IR (KBr) $v_{\max } 3360,2927$, 2877, 1630, 1455, 1382, 1073, 1041, $894 \mathrm{~cm}^{-1} ;{ }^{1} \mathrm{H}-\mathrm{NMR}\left(\mathrm{C}_{5} \mathrm{D}_{5} \mathrm{~N}, 600 \mathrm{MHz}\right)$ and ${ }^{13} \mathrm{C}-\mathrm{NMR}\left(\mathrm{C}_{5} \mathrm{D}_{5} \mathrm{~N}\right.$, $150 \mathrm{MHz}$ ) spectroscopic data-see Table 7; ESI-Q-Orbitrap MS m/z $1225.61841[\mathrm{M}-\mathrm{H}]^{-}$(calcd. for $\left.\mathrm{C}_{58} \mathrm{H}_{97} \mathrm{O}_{27}, 1225.62117\right)$.

Notoginsenoside $N L-C_{1}$ (8): White powder; $[\alpha]_{\mathrm{D}}^{25}-6.7$ (conc. 0.21, MeOH); UV $\lambda_{\max }(\mathrm{MeOH}) \mathrm{nm}$ (log $\varepsilon) 218$ (3.57); IR (KBr) $v_{\max }$ 3387, 2941, 2877, 1669, 1632, 1456, 1384, 1077, $1039 \mathrm{~cm}^{-1},{ }^{1} \mathrm{H}-\mathrm{NMR}$ $\left(\mathrm{C}_{5} \mathrm{D}_{5} \mathrm{~N}, 500 \mathrm{MHz}\right)$ and ${ }^{13} \mathrm{C}-\mathrm{NMR}\left(\mathrm{C}_{5} \mathrm{D}_{5} \mathrm{~N}, 125 \mathrm{MHz}\right)$ spectroscopic data-see Table 8; ESI-Q-Orbitrap MS m/z $929.51184[\mathrm{M}-\mathrm{H}]^{-}$(calcd. for $\mathrm{C}_{47} \mathrm{H}_{77} \mathrm{O}_{18}, 929.51044$ ).

Notoginsenoside $N L-C_{2}(9)$ : White powder; $[\alpha]_{\mathrm{D}}^{25}+1.8$ (conc. $\left.0.45, \mathrm{MeOH}\right) ; \mathrm{UV} \lambda_{\max }(\mathrm{MeOH}) \mathrm{nm}$ $(\log \varepsilon) 219$ (3.63); IR (KBr) $v_{\max }$ 3415, 2943, 2877, 1671, 1639, 1458, 1386, 1078, 1038, $894 \mathrm{~cm}^{-1} ;{ }^{1} \mathrm{H}-\mathrm{NMR}$ $\left(\mathrm{C}_{5} \mathrm{D}_{5} \mathrm{~N}, 500 \mathrm{MHz}\right)$ and ${ }^{13} \mathrm{C}-\mathrm{NMR}\left(\mathrm{C}_{5} \mathrm{D}_{5} \mathrm{~N}, 125 \mathrm{MHz}\right)$ spectroscopic data-see Table 9; ESI-Q-Orbitrap MS $m / z 929.51221[\mathrm{M}-\mathrm{H}]^{-}$(calcd. for $\mathrm{C}_{47} \mathrm{H}_{77} \mathrm{O}_{18}, 929.51044$ ).

Notoginsenoside $\mathrm{NL}-\mathrm{C}_{3}$ (10): White powder; $[\alpha]_{\mathrm{D}}^{25}+1.6$ (conc. 0.51, MeOH); UV $\lambda_{\max }(\mathrm{MeOH})$ $\mathrm{nm}(\log \varepsilon) 218$ (3.69); IR (KBr) $v_{\max } 3391,2942,2881,1663,1645,1453,1385,1164,1077,1040,895$ $\mathrm{cm}^{-1} ;{ }^{1} \mathrm{H}-\mathrm{NMR}\left(\mathrm{C}_{5} \mathrm{D}_{5} \mathrm{~N}, 500 \mathrm{MHz}\right)$ and ${ }^{13} \mathrm{C}-\mathrm{NMR}\left(\mathrm{C}_{5} \mathrm{D}_{5} \mathrm{~N}, 125 \mathrm{MHz}\right)$ spectroscopic data-see Table 10; ESI-Q-Orbitrap MS m/z 1091.56445 [M - H] $]^{-}$(calcd. for $\mathrm{C}_{53} \mathrm{H}_{87} \mathrm{O}_{23}, 1091.56327$ ).

Notoginsenoside NL-D (11): White powder; $[\alpha]_{\mathrm{D}}^{25}+29.6$ (conc. 0.34, MeOH); IR (KBr) $v_{\max } 3382$, 2953, 2878, 1717, 1649, 1456, 1387, 1312, 1075, 1048, $1008 \mathrm{~cm}^{-1} ;{ }^{1} \mathrm{H}-\mathrm{NMR}\left(\mathrm{C}_{5} \mathrm{D}_{5} \mathrm{~N}, 500 \mathrm{MHz}\right)$ and ${ }^{13} \mathrm{C}-\mathrm{NMR}\left(\mathrm{C}_{5} \mathrm{D}_{5} \mathrm{~N}, 125 \mathrm{MHz}\right)$ spectroscopic data-see Table 11; ESI-Q-Orbitrap MS m/z 855.47388 [M $\mathrm{H}]^{-}$(calcd. for $\mathrm{C}_{44} \mathrm{H}_{71} \mathrm{O}_{16}, 855.47366$ ).

Acid Hydrolysis of 1-11: Compounds 1-11 were hydrolyzed by acid and an aqueous layer was obtained by using the method reported in [12] —-their solution (each $2.0 \mathrm{mg})$ in $1 \mathrm{M} \mathrm{HCl}(1.0 \mathrm{~mL})$ was heated under reflux for $3 \mathrm{~h}$, respectively. Then, each reaction mixture was neutralized with Amberlite IRA-400 (OH- form) and removed by filtration. Next, the aqueous layer was subjected to the HPLC analysis under the following conditions-HPLC column, Kaseisorb LC NH$~_{2}-60-5,4.6 \mathrm{~mm}$ i.d. $\times 250$ $\mathrm{mm}$ (Tokyo Kasei Co. Ltd., Tokyo, Japan); detection and optical rotation [Chiralyser (IBZ Messtechnik GMBH, Mozartstrasse 14-16 D-30173 Hannover, Germany)]; a mobile phase, $\mathrm{CH}_{3} \mathrm{CN}_{-} \mathrm{H}_{2} \mathrm{O}(80: 20, v / v)$; and a flow rate of $0.7 \mathrm{~mL} / \mathrm{min}$. Then, D-xylose (from 2, 3, 6, 7, 9, and 10), L-arabinose (from 1, 3-6, 8, 
and 11), and D-glucose (from 1-11) and were confirmed by comparison of the retention times with the authentic samples $\left[t_{\mathrm{R}}: 9.4 \mathrm{~min}\right.$ (D-xylose), $10.3 \mathrm{~min}$ (L-arabinose), and $12.4 \mathrm{~min}$ (D-glucose), all of them showed positive optical rotations].

\subsection{Experimental Procedures for Bioassay}

The MTT and nitrite levels measurements of 1-11, as well as statistical analyses were conducted by using the method reported previously [16].

\section{Conclusions}

It is well-known that PNS are the major bioactive saponins in P. notoginseng. They were chemically classified as the derivatives of 20(S)-protopanaxatriol (PPT) and 20(S)-protopanaxadiol (PPD). In the present study, eleven new dammarane-type triterpenoid saponins were obtained and identified from the leaves extract of $P$. notoginseng. Among them, 1-10 were PPD-type saponins. It was suggested that PPD derivatives were the major PNS in P. notoginseng leaves, which was in accordance with the results found by other researchers [6-9]. As PPTs were reported to be the main PNS in P. notoginseng roots [17], we could not draw the conclusion that $P$. notoginseng leaves would be a possible replacement of the roots used in clinics.

From a structural point of view, the aglycones of compounds 1-10 were dammarane-type with oxygenated C-17 side chains different to protopanaxadiol. Compounds 1-4, 5-7, and 8-10 featured 23-ene25-hydroperoxyl, 23-ene-25-hydroxyl, and $\alpha, \beta$-unsaturated ketone, respectively. Among them, 23-ene-25-hydroperoxyl-substituted protopanaxadiol saponins were found in P. notoginseng leaves, first though they were found in P. notoginseng flowers [18], roots [19], and rhizomes [20]. Additionally, compounds 4 and 11 were characterized by the malonyl substitution at 3-position. The 3-malonyl substituted dammarane-type terpennoids are reported here for the first time. These results enrich the chemical investigations of $P$. notoginseng leaves.

Inflammation is widespread in the clinical pathology and closely associated to the progress of many diseases. NO is implicated in a variety of inflammatory conditions, which indicates the agents block NO production might be beneficial for the treatment of inflammatory responses. In this study, pretreatment of notoginsenosides NL-A 1 (1), NL-A 2 (2), NL-B 1 (5), NL-B 2 (6), NL-C $1-N L-C_{3}(8-10)$ at noncytotoxic concentration $(50 \mu \mathrm{M})$ decreased the NO production significantly, which indicated that they have an anti-inflammatory activity. Moreover, the inhibitory effects of all of them were found to be dose-dependent. Further investigations on their anti-inflammatory mechanisms would be beneficial.

Supplementary Materials: Supplementary data (The NMR and HRESIMS spectra of compounds 1-11, cell viability assay, as well as the raw data for western blot assays) associated with this article can be found online.

Author Contributions: Y.Z. (Yi Zhang) and T.W. designed the research and wrote the manuscript; F.S. and J.R. performed the experimental work; W.Z., Y.Z. (Ying Zhang), J.Y., and M.H. corrected the data and reviewed literatures; G.X. collected the plant sample; L.W. perfected the language. All authors have read and agreed to the published version of the manuscript.

Funding: This work was financially supported by grants from Important Drug Development Fund, Ministry of Science and Technology of China (2018ZX09711001-009-010, 2018ZX09735002).

Conflicts of Interest: The authors declare no conflict of interest.

\section{References}

1. Zhang, Z.; Chen, L.; Cui, X.; Zhang, Y.; Hu, Y.; Wang, C.; Xiong, Y. Identification of anti-inflammatory components of raw and steamed Panax notoginseng root by analyses of spectrum-effect relationship. RSC Adv. 2019, 9, 17950-17958. [CrossRef]

2. Xu, C.; Wang, W.; Wang, B.; Zhang, T.; Cui, X.; Pu, Y.; Li, N. Analytical methods and biological activities of Panax notoginseng saponins: Recent trends. J. Ethnopharmacol. 2019, 236, 443-465. [CrossRef] [PubMed]

3. Xu, Y.; Tan, H.Y.; Li, S.; Wang, N.; Feng, Y. Panax notoginseng for inflammation-related chronic diseases: A review on the modulations of multiple pathways. Am. J. Chin. Med. 2018, 46, 971-996. [CrossRef] [PubMed] 
4. Chen, Z.; Yang, L.; Wang, Y.; Yang, J.; Wei, M.; Huang, T.; Wang, B. Research advances in cultivation of Panax notoginseng. Wenshan Xueyuan Xuebao 2012, 25, 1-12. (In Chinese)

5. Cao, J.L.; Ma, L.J.; Wang, S.P.; Deng, Y.; Wang, Y.T.; Li, P.; Wan, J.B. Comprehensively qualitative and quantitative analysis of ginsenosides in Panax notoginseng leaves by online two-dimensional liquid chromatography coupled to hybrid linear ion trap Orbitrap mass spectrometry with deeply optimized dilution and modulation system. Anal. Chim. Acta 2019, 1079, 237-251. [PubMed]

6. Liu, X.Y.; Wang, S.; Li, C.J.; Ma, J.; Chen, F.Y.; Peng, Y.; Wang, X.L.; Zhang, D.M. Dammarane-type saponins from the leaves of Panax notoginseng and their neuroprotective effects on damaged SH-SY5Y cells. Phytochemistry 2018, 145, 10-17. [CrossRef] [PubMed]

7. Li, D.; Cao, J.; Bi, X.; Xia, X.; Li, W.; Zhao, Y. New dammarane-type triterpenoids from the leaves of Panax notoginseng and their protein tyrosine phosphatase $1 \mathrm{~B}$ inhibitory activity. J. Ginseng Res. 2014, 38, $28-33$. [CrossRef] [PubMed]

8. Huang, J.W.; Du, Y.Q.; Li, C.J.; Yang, J.Z.; Ma, J.; Zang, Y.D.; Chen, N.H.; Zhang, D.M. Neuroprotective triterpene saponins from the leaves of Panax notoginseng. Nat. Prod. Res. 2019, 22, 1-7. [CrossRef] [PubMed]

9. Huang, J.W.; Chen, F.Y.; Li, C.J.; Yang, J.Z.; Ma, J.; Chen, X.G.; Zhang, D.M. Two new saponins from the leaves of Panax notoginseng. J. Asian Nat. Prod. Res. 2018, 20, 337-343. [CrossRef] [PubMed]

10. Liu, F.; Ma, N.; Xia, F.B.; Li, P.; He, C.; Wu, Z.; Wan, J.B. Preparative separation of minor saponins from Panax notoginseng leaves using biotransformation, macroporous resins, and preparative high-performance liquid chromatography. J. Ginseng Res. 2019, 43, 105-115. [CrossRef] [PubMed]

11. Ahujaa, A.; Kim, M.Y.; Cho, J.Y. Protium javanicum Burm. methanol extract attenuates LPS-induced inflammatory activities in macrophage-like RAW264.7 cells. Evid. Based Complement. Alternat. Med. 2019, 2019, 2910278. [CrossRef] [PubMed]

12. Qu, L.; Wang, J.; Ruan, J.; Yao, X.; Huang, P.; Wang, Y.; Yu, H.; Han, L.; Zhang, Y.; Wang, T. Spirostane-type saponins obtained from Yucca schidigera. Molecules 2018, 23, 167. [CrossRef] [PubMed]

13. Yin, F.; Hu, L.; Lou, F.; Pan, R. Dammarane-type glycosides from Gynostemma pentaphyllum. J. Nat. Prod. 2004, 67, 942-952. [CrossRef] [PubMed]

14. Qiu, F.; Ma, Z.Z.; Xu, S.; Yao, X.S.; Chen, Y.J.; Che, Z.T. Studies on dammarane-type saponins in the flower-buds of Panax ginseng C.A. Meyer. J. Asian Nat. Prod. Res. 1998, 1, 119-123. [CrossRef] [PubMed]

15. Sun, H.; Ye, Y.; Pan, Y. Immunological-adjuvant saponins from the roots of Panax notoginseng. Chem. Biodivers. 2005, 2, 510-515. [CrossRef]

16. Ruan, J.; Li, Z.; Zhang, Y.; Chen, Y.; Liu, M.; Han, L.; Zhang, Y.; Wang, T. Bioactive constituents from the roots of Eurycomalongifolia. Molecules 2019, 24, 3157. [CrossRef]

17. Xia, P.; Zhang, S.; Liang, Z.; Qi, Z. Research history and overview of chemical constituents of Panax notoginseng. Zhongcaoyao 2014, 45, 2564-2570. (In Chinese)

18. Wang, J.R.; Yamasaki, Y.; Tanaka, T.; Kouno, I.; Jiang, Z.H. Dammarane-type triterpene saponins from the flowers of Panax notoginseng. Molecules 2009, 14, 2087-2094. [CrossRef] [PubMed]

19. Yoshikawa, M.; Murakami, T.; Ueno, T.; Hirokawa, N.; Yashiro, K.; Murakami, N.; Yamahara, J.; Matsuda, H.; Saijoh, R.; Tanaka, O. Bioactive saponins and glycosides. IX. Notoginseng (2): Structures of five new dammarane-type triterpene oligoglycosides, notoginsenosides-E, -G, -H, -I, and-J, and a novel acetylenic fatty acid glycoside, notoginsenic acid $\beta$-sophoroside, from the dried root of Panax notoginseng (Burk.) F. H. Chen. Chem. Pharm. Bull. 1997, 45, 1056-1062.

20. Song, J.P.; Zeng, J.; Cui, X.M.; Dai, Y.; Jiang, Z.Y.; Zhang, X.M.; Zhou, J.M.; Ma, Y.B.; Chen, J.J. Studies on chemical constituents from rhizomes of Panax notoginseng (II). Yunnan DaxueXuebao 2007, 29, 287-290. (In Chinese)

Sample Availability: Samples of the compounds 5, 8, 9, and 10 are available from the authors. 